\title{
Coexistence and Dynamical Connections between Hyperchaos and Chaos in the 4D Rössler System: A Computer-Assisted Proof*
}

\author{
Daniel Wilczak ${ }^{\dagger}$, Sergio Serrano ${ }^{\ddagger}$, and Roberto Barrio ${ }^{\ddagger}$
}

\begin{abstract}
It has recently been reported [P. C. Reich, Neurocomputing, 74 (2011), pp. 3361-3364] that it is quite difficult to distinguish between chaos and hyperchaos in numerical simulations which are frequently "noisy." For the classical four-dimensional (4D) Rössler model [O. E. Rössler, Phys. Lett. A, 71 (1979), pp. 155-157] we show that the coexistence of two invariant sets with different nature (a global hyperchaotic invariant set and a chaotic attractor) and heteroclinic connections between them give rise to long hyperchaotic transient behavior, and therefore it provides a mechanism for noisy simulations. The same phenomena is expected in other 4D and higher-dimensional systems. The proof combines topological and smooth methods with rigorous numerical computations. The existence of (hyper)chaotic sets is proved by the method of covering relations [P. Zgliczyński and M. Gidea, J. Differential Equations, 202 (2004), pp. 32-58]. We extend this method to the case of a nonincreasing number of unstable directions which is necessary to study hyperchaos to chaos transport. The cone condition [H. Kokubu, D. Wilczak, and P. Zgliczyński, Nonlinearity, 20 (2007), pp. 2147-2174] is used to prove the existence of homoclinic and heteroclinic orbits between some periodic orbits which belong to both hyperchaotic and chaotic invariant sets. In particular, the existence of a countable infinity of heteroclinic orbits linking hyperchaos with chaos justifies the presence of long transient behavior.
\end{abstract}

Key words. chaos, hyperchaos, computer-assisted proofs, covering relations, hyperchaotic saddle

AMS subject classifications. 37M25, 37C29, 37C70, 37B10, 65P20

DOI. $10.1137 / 15 \mathrm{M} 1039201$

1. Introduction. One of the most famous mathematical discoveries of the 20th century was the establishment of chaos theory. Chaotic systems are highly sensitive to initial conditions, having at least one direction of exponential spreading, while hyperchaotic behavior is characterized by two or more spreading directions [44]. Due to the variety of practical fields with models experiencing (hyper)chaotic behavior, many investigations have been conducted to shed light on the analysis of these dynamical systems. In recent years, several computerassisted methods for proving the existence of chaotic dynamics for maps and flows have been developed. They are often based on some topological tools like the Conley index [36, 39, 40] or the Brouwer degree $[60,63]$ or smooth ones like the shadowing theorem [27]. In these ap-

\footnotetext{
* Received by the editors September 10, 2015; accepted for publication (in revised form) by J. van den Berg December 21, 2015; published electronically February 18, 2016.

http://www.siam.org/journals/siads/15-1/M103920.html

${ }^{\dagger}$ Faculty of Mathematics and Computer Science, Jagiellonian University, Łojasiewicza 6, 30-348 Kraków, Poland (Daniel.Wilczak@ii.uj.edu.pl). The work of this author was partially supported by the Polish National Science Center under Maestro grant 2014/14/A/ST1/00453.

‡Departamento de Matemática Aplicada and IUMA, Computational Dynamics Group, University of Zaragoza, E50009 Zaragoza, Spain (sserrano@unizar.es, rbarrio@unizar.es). The work of these authors was supported by Spanish Research projects MTM2012-31883 and MTM2015-64095-P, the University of Zaragoza/CUD project UZCUD2015CIE-05, and the European Social Fund and Diputación General de Aragón (grant E48).
} 
proaches, chaos is understood as a symbolic dynamics embedded in the full dynamics. Based on theoretical results of the 1970s [3, 24], Tucker [48] was able to perform a computer-assisted proof (CAP) of the existence of the Lorenz chaotic attractor and its mathematical structure (the 14th Smale problem for the 21st century). In that paper a careful study of a normal form field around the equilibrium combined with global analysis of the Poincaré map and the cone field were used to prove that the Lorenz system admits a strange attractor which is also a support of the unique Sinai-Ruelle-Bowen measure for the flow.

Another well-known dynamical system which exhibits complicated dynamics is the threedimensional Rössler system [45] that is given by the system of ordinary differential equations (ODEs)

$$
\left\{\begin{array}{l}
\dot{x}=-y-z, \\
\dot{y}=x+b y, \\
\dot{z}=b+z(x-a) .
\end{array}\right.
$$

Zgliczyński [60] proved that, for the original parameter values given by Rössler, i.e., $a=$ $5.7, b=0.2$, a suitable Poincaré map is semiconjugated to the symbolic dynamics with a nontrivial invariant set. Later, Pilarczyk [39, 40] gave a computer-assisted proof that, for parameter values $a=2.2$ and $a=3.1$, several periodic orbits exist. Very recently, using the method based on the Lyapunov-Schmidt reduction and the rigorous integration of the third order variational equations it was proved [57] that, in the range of parameter values $a \in[2.83244,3.837358168411]$, there exist two period doubling bifurcations connected by a branch of period two (with respect to the Poincaré map) orbits. Besides, detailed numerical studies have been done recently $[7,8]$ showing the global parametric phase space structure.

In the literature most of the computer-assisted proof results have been stated for threedimensional models. However, many practical problems (for example, in chemical systems, neurology, electronic circuits, weather prediction, networks, and cryptography $[13,16,19,22$, $34,41,46,47])$ have to be modeled by dynamical systems with more than three dimensions, and some of them experience hyperchaotic behavior, a situation that cannot be given with dimension three. That is why a detailed study of four-dimensional (4D) and higher-dimensional dynamical systems is required, as some tools of analysis for three-dimensional chaotic models are no longer valid. Besides, the existence of more than one direction of spreading allows the system to undergo a broader spectrum of bifurcations. Therefore, the analysis of hyperchaotic systems is more complicated, and the numerical study of these systems may be, in some cases, not clear at all, giving quite frequently a confusing analysis about whether the system is hyperchaotic or it has a different behavior [42].

The main goal of this paper is to explain the "noisy" simulations obtained in many studies [42] and to give a CAP of some of the results about the coexistence of chaotic and hyperchaotic behavior that generates long transient time behavior and, so, an evident difficulty for numerical simulations. These transient behaviors are related to the existence of saddle invariant sets. Chaotic saddles [30] are closed, bounded, nonattracting, chaotic invariant sets with a dense orbit such that each point of the set has a stable direction and at least one unstable direction. The skeleton that configures the invariant set is the infinite number of unstable periodic orbits embedded in the saddle [17]. In the hyperchaotic saddles there are an infinite number of unstable periodic orbits with two (or more) unstable directions. 
As a first step in the study in detail of that problem, we use another well-known system: the 4D Rössler system [44] given by

$$
\left\{\begin{array}{l}
\dot{x}=-(y+z), \\
\dot{y}=x+a y+w, \\
\dot{z}=b+x z \\
\dot{w}=-c z+d w .
\end{array}\right.
$$

This system was used to introduce the concept of hyperchaos. For a wide range of parameter values a certain Poincaré map of this system exhibits a strongly attracting invariant set with two expanding directions and one contracting direction (and so it is hyperchaotic). In [32] strong numerical evidence of the existence of symbolic dynamics on two symbols for the ninth iterate of this Poincaré map was presented. In [55] a CAP of the existence of chaotic dynamics and abundance of homoclinic and heteroclinic orbits between two periodic orbits with two expanding directions in the 4D Rössler system was presented. This result gives a mathematical proof of the existence of hyperchaotic behavior. The method utilizes topological tools of the covering relations [63] with the cone conditions [28]. We remark that the study of the existence of homoclinic or heteroclinic orbits is a much harder task than just verifying the existence of symbolic dynamics, since it requires some tools guaranteeing convergence.

Note that in $[12,33]$ a strong numerical evidence of the existence of hyperchaotic saddles in the 4D Rössler system was also presented, but the complete rigorous verification of assumptions of the main topological theorem by means of interval analysis has not been done previously in the literature.

The following list summarizes the main results concerning the system (2) proved in this paper using CAP techniques for an open set of parameter values $a=0.27857, b=3, c=0.3$, $d=0.05$ :

- There exists an explicitly given trapping region $\mathcal{B}$ (Theorem 4.1).

- There exist six periodic orbits (see Figure 1). The orbits corresponding to the points $p_{i}$ have a one-dimensional unstable invariant manifold, and those corresponding to points $q_{i}$ have a two-dimensional unstable invariant manifold (Theorem 4.2).

- The maximal invariant set in the trapping region $\mathcal{B}$ contains three invariant sets, say $S_{1}, S_{2}, S_{3}$, on which the dynamics is $\Sigma_{2}$ chaotic; i.e., it is semiconjugated to the Bernoulli shift on two symbols (Theorem 4.5 and Corollary 4.6).

- $S_{1}$ is a hyperchaotic set with two positive Lyapunov exponents (Theorem 4.5 and Corollary 4.6).

- $S_{2}$ and $S_{3}$ are chaotic sets with one positive Lyapunov exponent (Theorem 4.5 and Corollary 4.6).

- There is a countable infinity of periodic orbits of arbitrary large periods (every periodic path on the graph presented in Figure 1 is realized by a periodic orbit) (Theorem 4.5).

- There is a countable infinity of heteroclinic/homoclinic orbits linking periodic orbits inside each horseshoe, and a countable infinity of heteroclinic connections linking $S_{1}$ with $S_{2}, S_{2}$ with $S_{3}$, and $S_{1}$ with $S_{3}$. In fact, every finite and nonconstant path on the graph presented in Figure 1 is realized by a homoclinic/heteroclinic connection between periodic orbits at the beginning and at the end of this sequence (Theorem 4.5). 


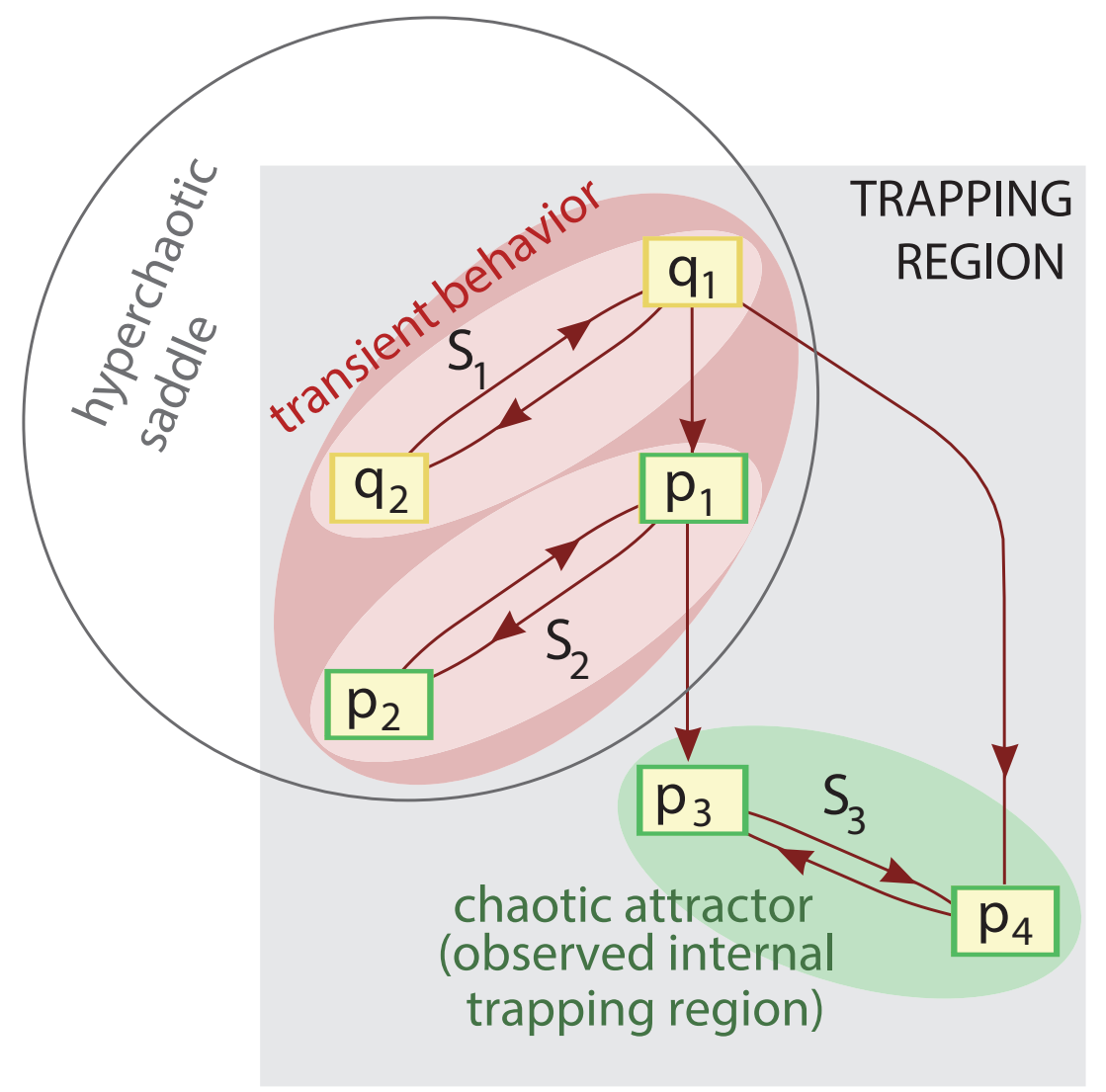

Figure 1. Visualization of the types of dynamics resulting from Theorems 4.1 and $4.5 . S_{1}$ is a hyperchaotic invariant set; $S_{2}, S_{3}$ are chaotic invariant sets. They are linked by heteroclinic orbits.

We would like to emphasize that in order to obtain the existence of dynamical linking between hyperchaos and chaos we had to extend the method of covering relations [63] to the case of a nonincreasing number of unstable directions - Theorem 3.3. This is the main theoretical result of this paper that can be applied to study other, perhaps higher-dimensional systems.

The above mentioned list of results concerning the Rössler system is a clear indication that such phenomena occur in other 4D and higher-dimensional models.

This paper is organized as follows. In section 2 we present several numerical simulations showing technical difficulties in locating different kinds of behaviors and attractors in the 4D Rössler system, and we also show the global existence of a hyperchaotic invariant set. In section 3 we recall the method of covering relations [63] and several computational topological tools to make this paper more self-consistent. There we also prove Theorem 3.3, which is a theoretical tool for numerical validation of the existence of heteroclinic connections between periodic orbits of different types. In particular, it is used to prove the existence of a dynamical link between hyperchaos and chaos in the $4 \mathrm{D}$ Rössler system. In section 4 we present the main theorem about the 4D Rössler system, and in section 5 we give details of the CAP of the coexistence of chaos and hyperchaos in the Rössler system.

We note that the existence of chaotic/hyperchaotic dynamics and abundance of homo- 
clinic and heteroclinic orbits is proved by means of interval analysis. The proof requires the computation of rigorous bounds for a Poincaré map together with its derivatives. The $\mathrm{C}++$ program which realizes the necessary computations is available in the accompanying supplementary material (M103920_01.zip [local/web 8.49MB]) and from [53].

2. Chaos and hyperchaos. In this section we present several numerical simulations that show the coexistence of various types of behaviors in $4 \mathrm{D}$ differential systems, and we show some of the difficulties in the numerical simulations. We will use, as a paradigmatic example, the well-known 4D Rössler model [44], given by (2), where we fix the values of parameters $b=3.0$ and $d=0.05$, and we allow changing the values of $a$ and $c$.

In all the numerical simulations we use a Taylor series method as the numerical ODE integrator (the free software TIDES $[1,2]$ ) with variable order and stepsize. Note that this method is quite robust and accurate, and it permits us to obtain multiple-precision results if needed [10].

Our first question is related to the computational approach to classifying the different behaviors. In numerical simulations a common way to detect chaotic/hyperchaotic behavior is by calculating the Lyapunov spectra [4] of an orbit. If one of the Lyapunov exponents is positive, the orbit exhibits sensitive dependence on initial conditions, and this is a standard indication of chaotic behavior, while in the case of hyperchaotic behavior two (or more) Lyapunov exponents have to be positive [44]. In Figure 2 we compute the Lyapunov spectra of the $4 \mathrm{D}$ Rössler model with the method of Wolf et al. [58] at the parameter line $c=0.31$ and changing $a \in[0.263,0.28]$. In the pictures on the top we show the dependence of the classification of the different detected behaviors in function on how we perform the numerical simulations. We consider five different approaches using different transient time $T_{t}$ (time interval before beginning to compute the Lyapunov exponents) and different integration time $T_{c}$ (real interval of computation of the exponents): $\mathrm{t} 1: T_{t}=0, T_{c}=3000 ; \mathrm{t} 2: T_{t}=3 \cdot 10^{4}$, $T_{c}=3000 ; \mathrm{t} 3: T_{t}=3 \cdot 10^{5}, T_{c}=3000 ; \mathrm{t} 4: T_{t}=3 \cdot 10^{4}, T_{c}=15000 ; \mathrm{t} 5: T_{t}=3 \cdot 10^{5}$, $T_{c}=15000$. Once we compute the complete Lyapunov spectra (in our case the four Lyapunov exponents) we use them to classify the different orbits. In the studied line we show in the figure with colored dots the different behaviors detected. A blue dot represents a limit cycle, with maximum Lyapunov exponent $\lambda_{1}=0$ and the others $\lambda_{2,3,4}<0$; red for torus, $\lambda_{1,2}=0$ and $\lambda_{3,4}<0$; green for chaos, $\lambda_{1}>0, \lambda_{2}=0$, and $\lambda_{3,4}<0$; orange for weak hyperchaos, $0.05>\lambda_{1}>\lambda_{2} \gtrsim 0, \lambda_{3}=0$, and $\lambda_{4}<0$; gray for strong hyperchaos, $\lambda_{1}>0.05>\lambda_{2}>0$, $\lambda_{3}=0$, and $\lambda_{4}<0$. Note that we have differentiated the cases of having two positive Lyapunov exponents (strong hyperchaos) with the case (weak hyperchaos) of having two positive values but one of them quite small, $\lambda_{2} \gtrsim 0$.

From the numerical simulations, we observe that the results have to be taken with care if only one simulation is done. We observe that a sufficient large transient time $T_{t}$ is necessary for a correct analysis of the behavior, and this is more important than taking too large integration time $T_{c}$. In this problem, and from our numerical tests, we observe that a good selection, which ensures a good numerical simulation and an affordable computing time, is $T_{t}=3 \cdot 10^{4}, T_{c}=15000$ (that is, option t4) if a large set of simulations has to be done. Note that other options, except $\mathrm{t} 5$, which gives slightly better results but with much more computational effort, give in some regions an incorrect identification of the behavior of the 


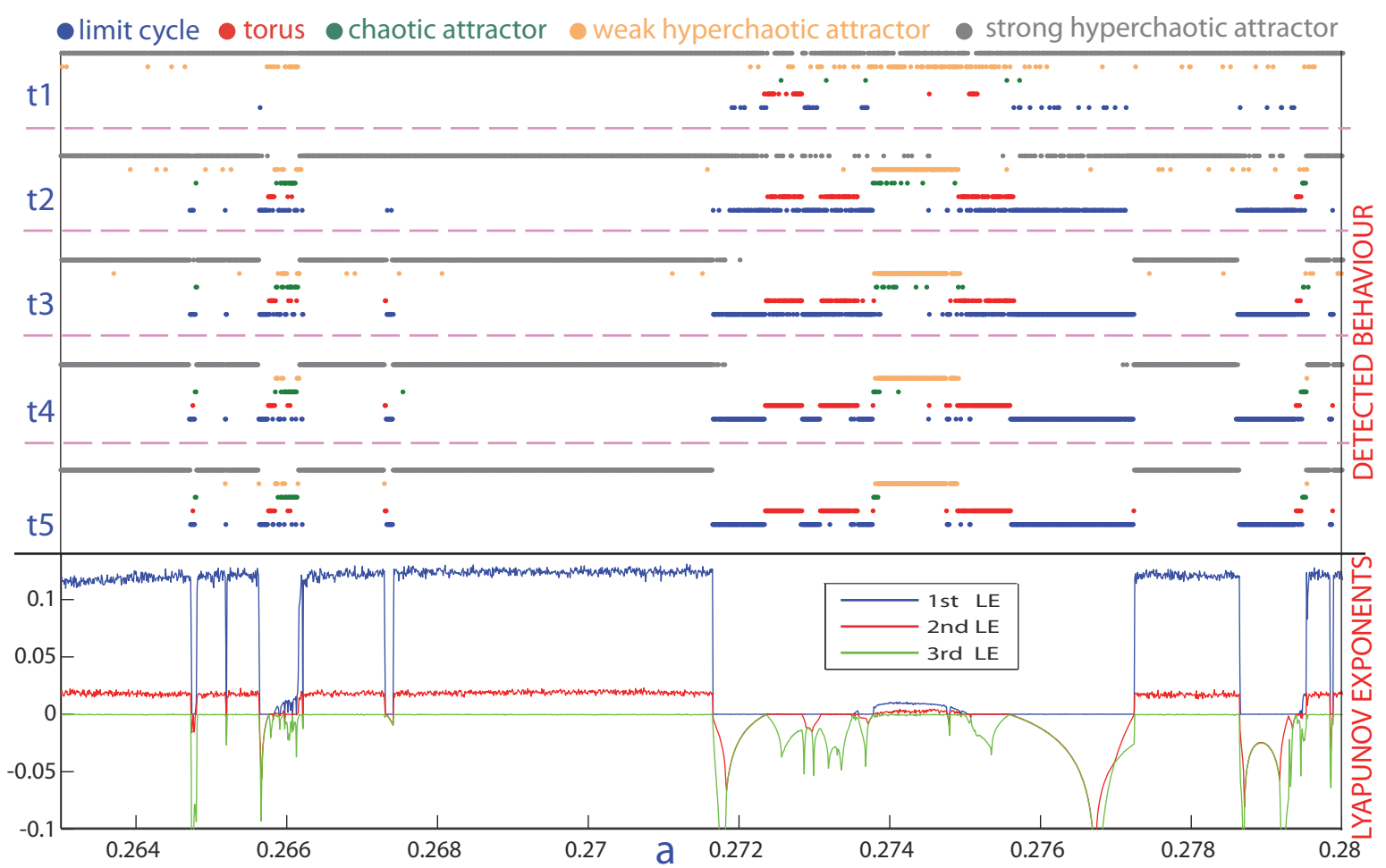

Figure 2. Top figures: Classification of detected behavior in function of Lyapunov exponents. Color code: blue: limit cycle; red: torus; green: chaotic; orange: weak hyperchaotic; and grey: strong hyperchaotic behavior. Each block uses in the computations different transient $\left(T_{t}\right)$ and integration $\left(T_{c}\right)$ times: t1: $T_{t}=0, T_{c}=3000$; $\mathrm{t} 2: T_{t}=3 \cdot 10^{4}, T_{c}=3000 ; \mathrm{t} 3: T_{t}=3 \cdot 10^{5}, T_{c}=3000 ; \mathrm{t} 4: T_{t}=3 \cdot 10^{4}, T_{c}=15000 ; \mathrm{t} 5: T_{t}=3 \cdot 10^{5}$, $T_{c}=15000$. Bottom: first three Lyapunov exponents computed using the t5 time environment.

system.

To see such a situation more clearly we show in Figure 3 two biparametric plots presenting all Lyapunov exponents. This type of parametric plots has proved to be quite useful in the location of different dynamical phenomena and global bifurcations in different systems like the location of spiral structures in dissipative systems related to some homoclinic bifurcations $[7,8,9,49]$, in the theoretical study of the chaotic region in mathematical neuron models [11], in the location of stable regions far away from the escape energy in the classical Hénon-Heiles Hamiltonian [6], and so on. Therefore, a biparametric plot has become a quite useful tool, and so it is of great interest to study the most suitable way to perform it, and also the reasons why in some circumstances we have to take care if we want to obtain suitable results. The only difference in the simulations in Figure 3 is the different values used for transient time, before, and integration time, during the computation of the Lyapunov exponents (cases t1 and t4 in Figure 2). As in Figure 2 we have differentiated the cases of having two large positive Lyapunov exponents (strong hyperchaos) with the case of having two positive values but one of them quite small (weak hyperchaos). The colors in the figure determine the different behaviors detected: white represents a stable limit cycle; blue torus; red chaos; green weak hyperchaos; and brown strong hyperchaos. Therefore, we observe that in the biparametric 

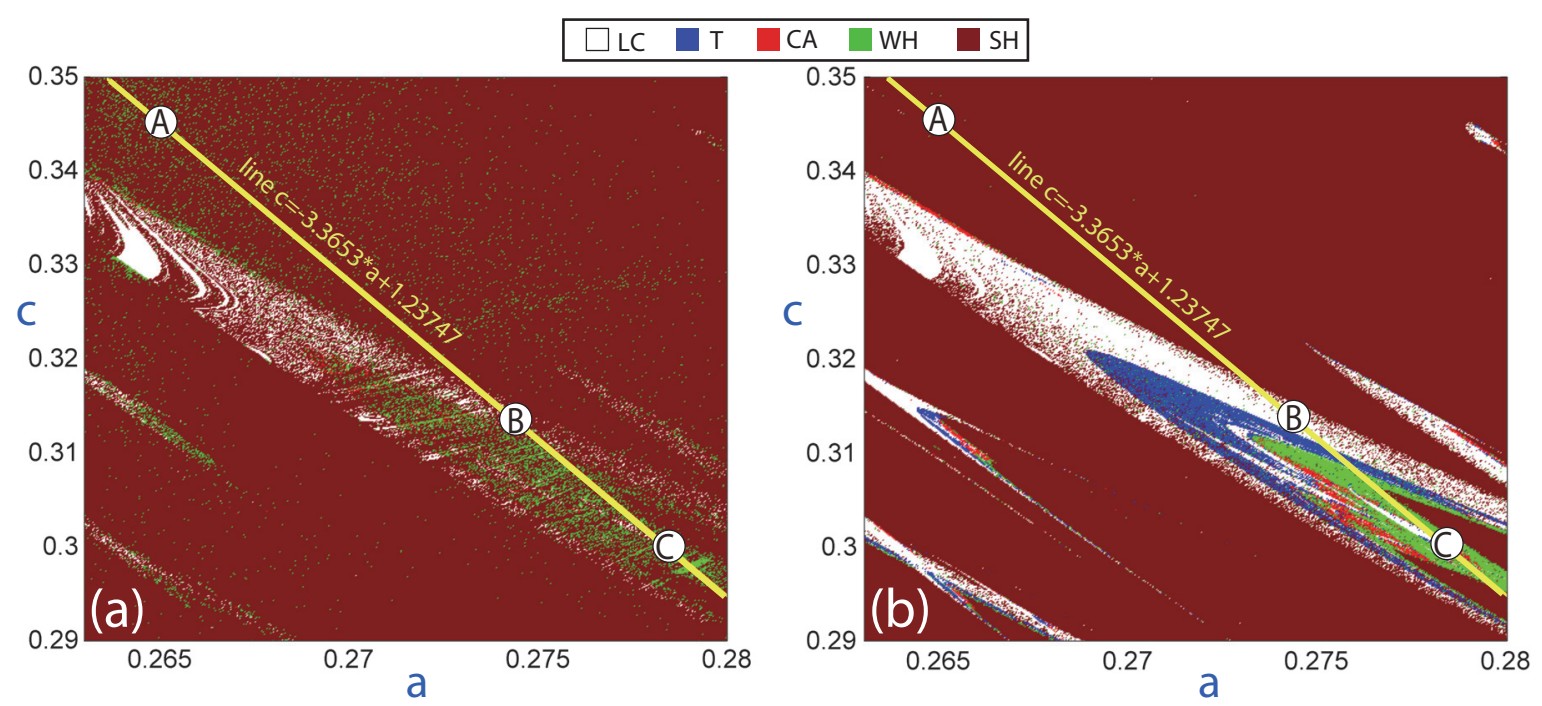

Figure 3. Lyapunov exponents presented by biparametric plots showing periodic (limit cycles, LC), quasiperiodic (torus, T), chaotic (CA), weak hyperchaotic (WH), and strong-hyperchaotic (SH) behaviors. (Left) $T_{t}=0, T_{c}=3000$. (Right) $T_{t}=3 \cdot 10^{4}, T_{c}=15000$.

plots a plethora of different behaviors are detected. Comparing both pictures, we can see how the left picture is completely dominated by brown, representing hyperchaotic behavior. Note that we have a "noisy" picture without giving a clear study of the real behavior. Even so, there are behaviors that are not detected. For example, the existence of torus is not signed throughout the study region (recently, it has been shown that the location of quasiperiodic behavior or torus is of practical interest [29]). This situation appears also in most of the simulations in the literature [42]. In contrast, in the right picture, wherein we have used a suitable transient time, those structures that were hardly visible in the left picture now appear in a clearer way and torus existence is correctly identified, but still some "noisy" patterns appear. The first question is whether the left picture, at least, correctly identifies the behavior of the system at the final integration time. In $[12,31,51]$ the authors show that algorithms to obtain Lyapunov exponents need some time before they detect the change between the transient and the asymptotic behaviors. Therefore, if we want to classify the behavior of the system at a fixed time, we must arrive at its asymptotic behavior before starting the calculation of Lyapunov exponents. So, to classify the type of attractor which controls the dynamics of the system, we have to consider a sufficiently long transient time. We can see that the right picture of Figure 3 still shows "noise" so that higher transient and/or integration time is necessary to get an absolutely clear picture. But, higher values provide little improvement that is not worth the cost in computational time involved (note that using the values of the case t5, a simulation with $500 \times 500$ points may last 189 days on an Intel Core2 Duo E6750 @ 2.66GHz). This observation permits us to perform better simulations of Lyapunov exponents, but in any case it does not provide a complete explanation of the "noisy" patterns and why we need such a long transient time in this system. 
To study in detail what really happens, we focus our attention to a line,

$$
c(a)=-3.3653 \cdot a+1.23747,
$$

in the biparametric picture (the straight line in Figure 3) because it crosses regions with all the different behaviors detected in the model. In Figure 4 we show a bifurcation diagram obtained computing the Poincaré section at $\left\{(x, y, z, w) \in \mathbb{R}^{4} \mid \dot{y}=0, \ddot{y}>0\right\}$ and projecting the results on the variable $x$. Note that we observe some of the familiar routes to hyperchaos, like tangencies among the hyperchaotic saddle and a periodic orbit (fold bifurcations of limit cycles) or smooth growing of the second Lyapunov exponent, as pointed out in [26, 33]. On the bottom plots we show two projections of the attractors and the hyperchaotic saddle for three parametric value sets (the same sets outlined in Figure 3). In the first point, A, the system has one hyperchaotic attractor, whereas in the other two there are a hyperchaotic saddle coexisting with a limit cycle, B, or a chaotic attractor of small size, C. In fact, from the bifurcation diagram, we observe that close to the values of the parameters where the stable limit cycle exists, there appears a small chaotic attractor via the standard period doubling cascade, and in some cases it becomes smoothly hyperchaotic (weak hyperchaos) or it grows suddenly to become a large hyperchaotic attractor (strong hyperchaos). A remarkable fact is the global existence of a hyperchaotic invariant set along all the line. The existence of the hyperchaotic invariant, sometimes as a global attractor and sometimes as a saddle structure, is really observed along all the biparametric simulations of Figure 3. This invariant originates a long transient hyperchaotic behavior and hence "noisy" simulations because, depending on the transient time, we will only observe the global hyperchaotic transient behavior.

The existence of the hyperchaotic saddle in most of the parameter space gives rise to several options in the creation or destruction of chaotic or hyperchaotic attractors. From Figure 4 there is a clear tangent bifurcation in the disappearance of the large hyperchaotic attractor around $a \approx 0.2732$, maintaining later the hyperchaotic saddle and a stable limit cycle. Afterwards, a complicated region appears, where invariant torus, chaotic attractors, and small hyperchaotic attractors are created. In Figure 5 we study a magnification (zoom 1 area) of Figure 4. In this figure, we observe from the picture on the top of the Lyapunov exponents that the changes from torus, chaos, and hyperchaos are quite smooth and progressive, and the Lyapunov exponents grow smoothly. When two of the exponents reach the zero value, some regions of invariant torus are observed. Later, the first Lyapunov exponent grows, creating a chaotic attractor, but note as the third Lyapunov exponent is smoothly growing in most of the picture. When this value reaches a value close to zero, the second Lyapunov exponent grows also, giving rise to a hyperchaotic attractor whose Lyapunov dimension [21] grows smoothly (region with an interrogant in the picture denotes that the exact point where the hyperchaotic attractor is created cannot be identified accurately, as the change is quite progressive in this case). To explain this smooth change, several scenarios have been proposed in the literature. In $[26,59]$ the authors argue that the chaos-hyperchaos transition is mediated by changes in the stability of an infinite number of unstable periodic orbits (UPOs) embedded in the chaotic attractor changing from having one unstable direction to having two. This coexistence in the chaotic invariant set is responsible for the occurrence of nonhyperbolic behavior known as unstable dimension variability (UDV) [38]. The onset of UDV is usually related to the loss of transversal stability of an unstable low-period saddle embedded in the chaotic set, through a 


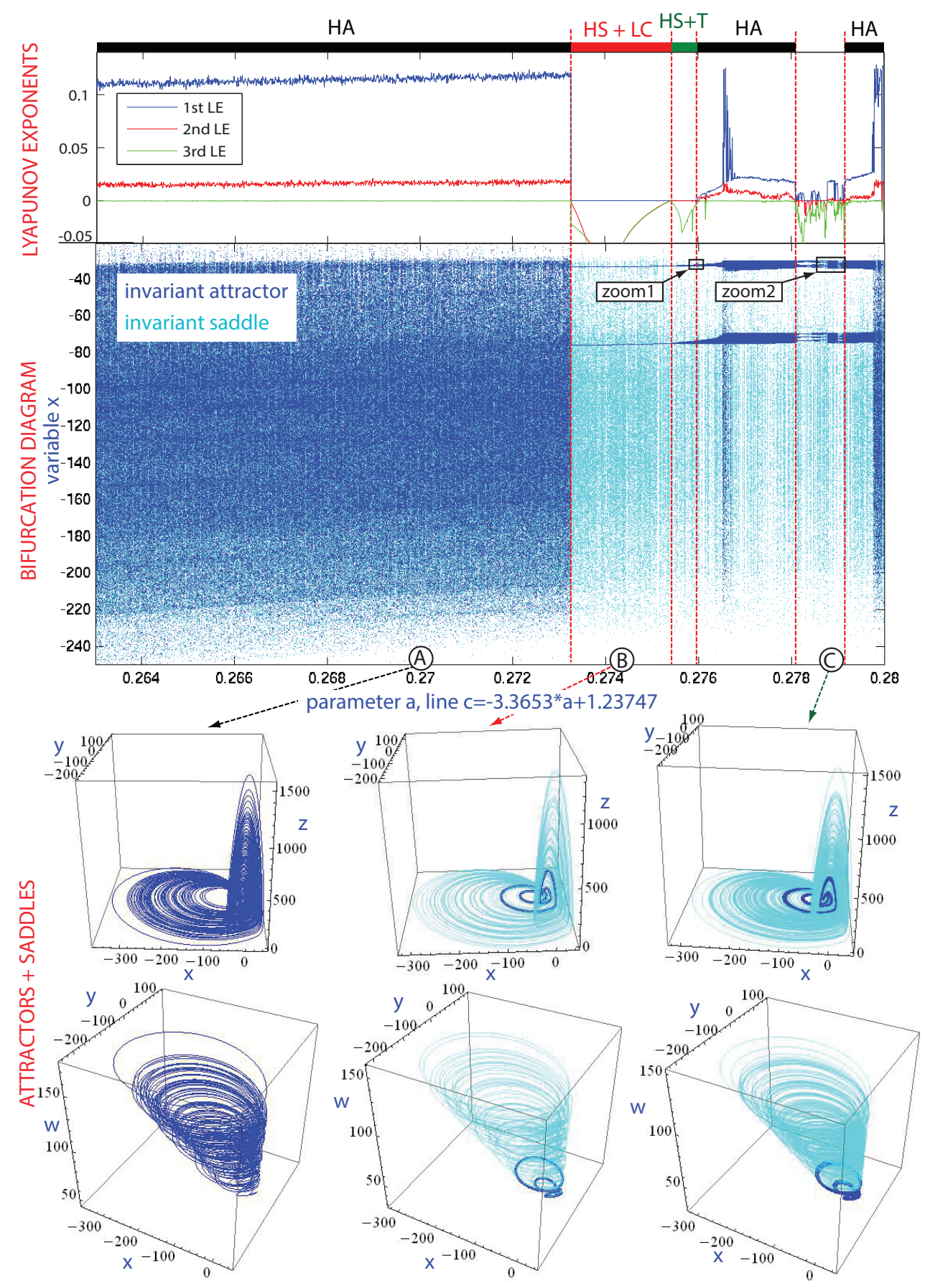

Figure 4. (Top) Lyapunov exponents showing periodic (limit cycles, LC), quasi-periodic (torus, T), chaotic attractor (CA), hyperchaotic attractor (HA), and hyperchaotic saddle (HS) behaviors along the selected line in the biparametric plot. (Middle) Bifurcation diagram projecting in variable $x$ showing the attractors and saddles. (Bottom) Projections of the different invariants (attractors and saddles) at three selected parameter values. 


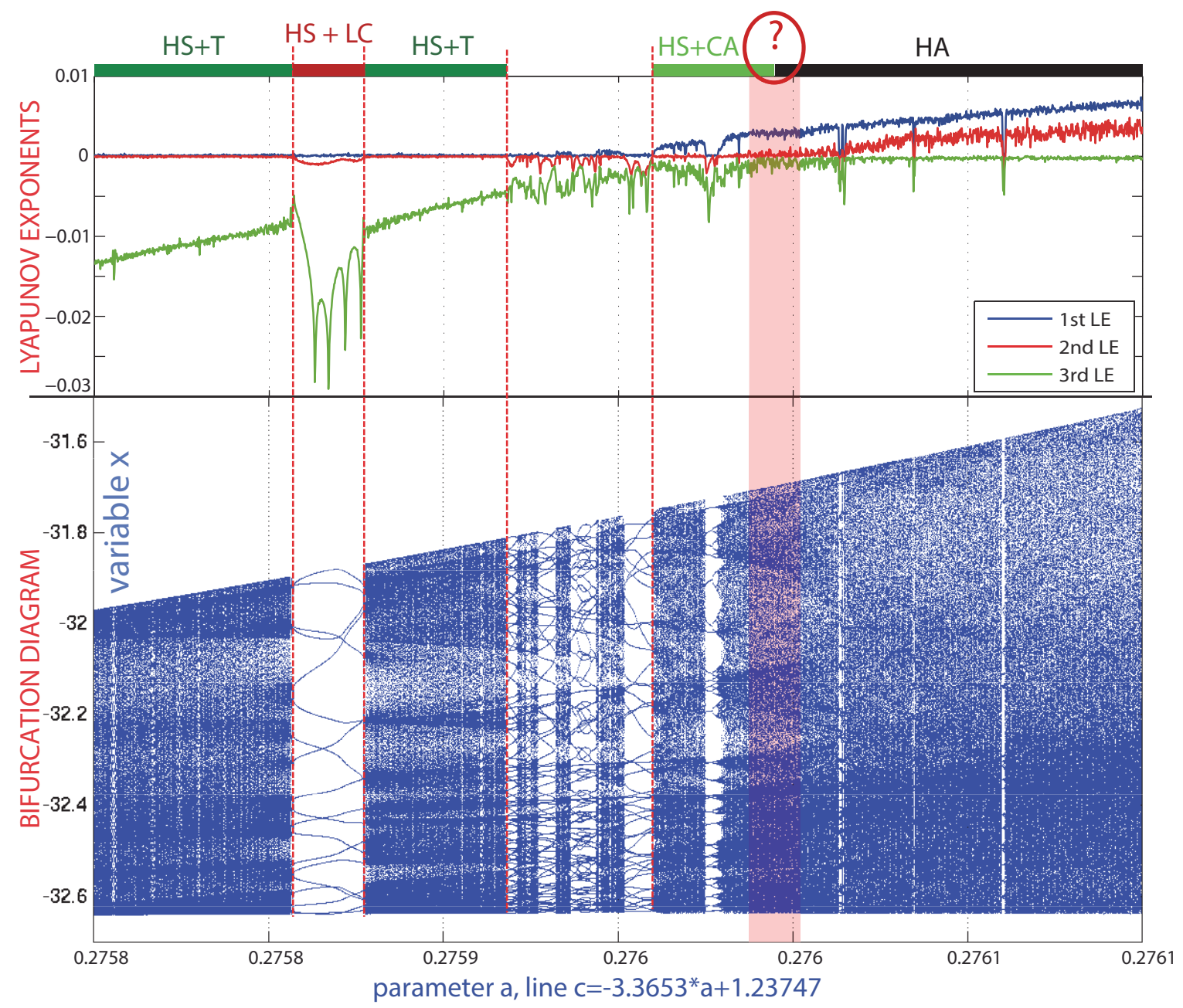

Figure 5. Zoom 1 in Figure 4. (Top) Lyapunov exponents showing periodic (limit cycles, LC), quasi-periodic (torus, T), chaotic attractor (CA), hyperchaotic attractor (HA), and hyperchaotic saddle (HS) behaviors along the selected line in the biparametric plot. (Bottom) Bifurcation diagram projecting in variable $x$ showing the attractors. The interrogation mark denotes the region where the hyperchaotic attractor is smoothly created.

local bifurcation. In this paper we give some CAPs (see the next sections) of the coexistence of these kinds of UPOs, and, moreover, we prove the existence of heteroclinic connections among them establishing chaotic and hyperchaotic invariant sets. The changes in the way these invariant sets are connected among them will give rise to the smooth changes in the parameter space. We argue that the smooth change is due not only to the change of the unstable directions of the UPOs embedded in the attractors but also in the inclusion by the attractor of new invariant sets that have more unstable directions and that belong to the hyperchaotic saddle.

Unlike what happens in Figure 5, in Figure 6 (zoom 2 area of the Figure 4) more sudden changes occur. Moreover, in recent years several papers have focused their attention on 


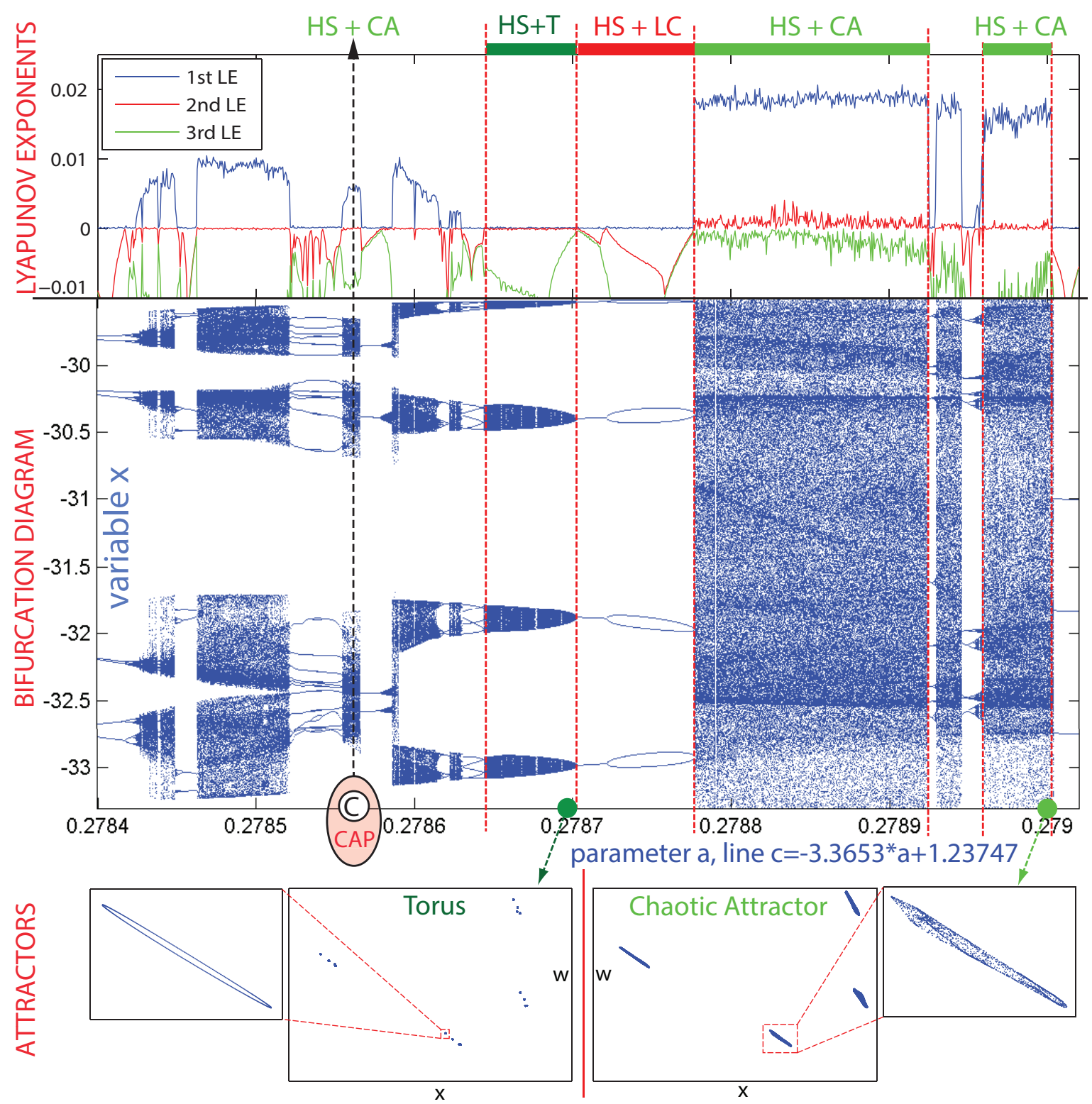

Figure 6. Zoom 2 in Figure 4. (Top) Lyapunov exponents showing periodic (limit cycles, LC), quasiperiodic (torus, T), chaotic attractor (CA), and hyperchaotic saddle (HS) behaviors along the selected line in the biparametric plot. (Middle) Bifurcation diagram projecting in variable $x$ showing the attractors. (Bottom) Projections of the invariant torus and the chaotic attractor filling the area of the torus at two selected parameter values. The point $C$ denotes the point where the CAP proof is done in the next sections.

the existence of quasi periodicity (invariant torus) in coupled and high-dimensional systems $[5,14,29]$. In fact, it is well known, from the seminal paper of Kaneko [25], that another route to chaos and hyperchaos is the torus breakdown. In [25] he proposed a scenario in twodimensional mappings where the torus appears via a Hopf bifurcation, the shape of the torus is 
distorted and regions of frequency lockings increase, chaos appears through a period doubling or a tangent bifurcation of some of the frequency-locked cycles, the Lyapunov dimension of the attractor increases, and later two Lyapunov exponents take positive values. Some of these phenomena are observed in the 4D Rössler model in Figure 6, where torus, chaotic attractors, and hyperchaotic saddles are presented in regions where smooth changes in the Lyapunov exponents are observed (due to changes in the UPOs foliated to the attractors or successive joinings of more UPOs) but also some sudden increases (due to the tangent bifurcations). The bifurcation diagram and the projections of the attractors at two parameter values in Figure 6 show that the chaotic attractors fill the region delimited by the invariant torus, as frequently shown in the literature for maps [25].

The values used by Rössler [44] ( $a=0.25, b=3, c=0.5$, and $d=0.05)$ to show the existence of hyperchaotic behavior and attractors are located in a region of robust hyperchaos (on the upper-left part of the biparametric plots of Figure 3). This situation helped the proof in [55] that the Poincaré map of the 4D Rössler model, for the previous values, exhibits chaotic dynamics with an attractor possessing two expanding directions. This was the first appearance in the literature demonstrating mathematically the existence of hyperchaotic behavior. However, as we have remarked, along the selected line we may observe a plethora of different behaviors. Where the hyperchaotic set is a saddle, it may coexist with a variety of other behaviors depending on the specific values of the parameters. It is in this context where we have selected one special point, $\mathrm{C}$, where the CAP is done in the next sections.

The above numerical study shows the apparent existence of invariant sets with different natures and how they generate the coexistence of regular, chaotic, and hyperchaotic behaviors. This situation, together with the hypothesis of the global existence of a hyperchaotic invariant set, forms a complete explanation of the difficulty of providing a clear study of the behavior of these systems. Therefore, the rest of this paper focuses its attention on providing some rigorous proofs of the existence of these behaviors at the selected parameter values via CAP techniques.

3. Covering relations with decreasing nominally unstable dimensions. In the last few years CAP techniques have been developed to study differentiable dynamical systems by introducing topological analogues of a Markov partition [63]. Note that, if obtained, a Markov partition represents a fundamental construction giving a rigorous coding to the orbits of the system (symbolic dynamics). To that goal, one strives to subdivide a particular region of the phase space into blocks which cross one another in a topologically consistent way under iteration. We have in mind to use suitable topological objects to show that the underlying dynamical system (in our case the 4D Rössler model) exhibits so-called topological horseshoe generalizations of Smale's horseshoe with two unstable directions coexisting with Smale's horseshoes with one unstable direction. That is, we want to prove the coexistence of hyperchaotic and chaotic behaviors. The presence of these horseshoes can be inferred from modeling the dynamics by linear maps, which indicate hyperbolic-like expansions and contractions.

In [63] the authors introduced the notion of covering relations - a topological tool for rigorous verification of the existence of topologically hyperbolic dynamics-like horseshoes. The method, together with the cone condition criterion [28], is a powerful method for rigorous validation of the existence of hyperbolic invariant sets and, in general, normally hyperbolic 
invariant sets $[20,52]$.

This notion has been extended previously in the literature to the case when the number of stable directions of so-called $h$-sets along the orbit is changing [54].

In this section we propose another extension of this method to the case when the observed nominally unstable dimensions are varying. This tool will be used to prove the existence of heteroclinic linking between hyperchaos and chaos. To this end, we will change the definition of covering relations from [63] slightly.

By $B_{n}$ we will denote a unit ball in the maximum norm, i.e., $B_{n}=[-1,1]^{n}$.

Definition 3.1. An $h$-set $N$ is a triple $N=\left(h_{N}, u, s\right)$, where

- $u, s$ are natural numbers that stand for the dimensions of nominally unstable and stable directions, respectively, and

- $h_{N}$ is a homeomorphism of $\mathbb{R}^{u+s}$.

An $h$-set is a geometric object, a compact subset of $\mathbb{R}^{u+s}$ that is homeomorphic to $B_{u+s}$ via the homeomorphism $h_{N}$. We will call this geometric realization the support of the $h$-set $N$, and we denote it by $|N|=h_{N}\left(B_{u+s}\right)$. The subsets of the boundary of $|N|$ defined by

$$
\begin{aligned}
& N^{-}=h_{N}\left(\partial B_{u} \times B_{s}\right), \\
& N^{+}=h_{N}\left(B_{u} \times \partial B_{s}\right)
\end{aligned}
$$

will be called exit and entrance sets, respectively. Although an $h$-set is just a geometric object, the meaning of $N^{ \pm}$will become clear when we will use this notion in a dynamical context.

Note that the above definition is equivalent to that from [63]. In the original definition the authors define an $h$-set as a set homeomorphic to the product of two unit balls $B_{u} \times B_{s}$ in some norm. Here we just fix the maximum norm which has the nice property that the Cartesian product of two balls in this norm is a ball in higher dimension, which simplifies further considerations. In the definition below we will use a natural identification of Cartesian products of sets with subsets of higher-dimensional spaces.

Definition 3.2. Let $N=\left(h_{N}, u_{N}, s_{N}\right)$ and $M=\left(h_{M}, u_{M}, s_{M}\right)$ be h-sets such that $u_{N} \geq$ $u_{M}$, and put $n=u_{N}+s_{N}, m=u_{M}+s_{M}$. Let $f:|N| \rightarrow \mathbb{R}^{m}$ be a continuous map. We say that the h-set $N f$-covers the h-set $M$, denoted by $N \stackrel{f}{\Longrightarrow} M$, if there is a homotopy $H:[0,1] \times B_{u_{M}} \times B_{n-u_{M}} \rightarrow \mathbb{R}^{m}$ and a linear map $L: \mathbb{R}^{u_{M}} \rightarrow \mathbb{R}^{u_{M}}$ such that

$$
\begin{aligned}
H(0, \cdot) & =h_{M}^{-1} \circ f \circ h_{N}, \\
H(1, x, y) & =(L \cdot x, 0) \in \mathbb{R}^{u_{M}} \times \mathbb{R}^{s_{M}}, \\
H(t, x, y) & \notin B_{m} \text { for } t \in[0,1], \quad(x, y) \in \partial B_{u_{M}} \times B_{n-u_{M}}, \\
H(t, x, y) & \notin B_{u_{M}} \times \partial B_{s_{M}} \text { for } t \in[0,1], \quad(x, y) \in B_{n} .
\end{aligned}
$$

The geometry of this definition is shown in Figure 7 (see also Figures 16 and 17 for its realization into the 4D Rössler model). Observe that the above conditions imply that $L$ must be an expanding isomorphism with all eigenvalues out of the unit disc. If $u_{M}=u_{N}$, then this definition coincides with that from [63] given just for a constant number of unstable directions.

The following theorem extends the applicability of the method of covering relations to the case when the number of observed nominally unstable directions might be decreasing along trajectories. Generically, we cannot get recurrence in this case, but it is still possible to obtain 

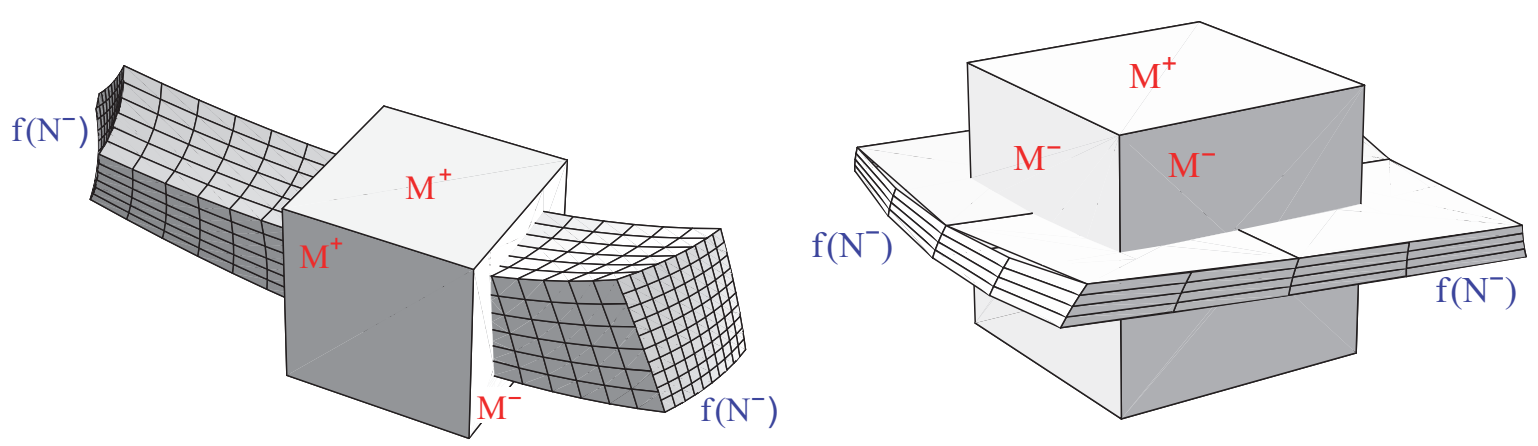

Figure 7. Illustration of covering relations with constant number of unstable directions (left) $u_{M}=u_{N}=1$ and (right) $u_{M}=u_{N}=2$. The image of entire set $|N|$ is mapped across the set $|M|$ without touching its entrance set $M^{+}$. Thus, it can be deformed by a homotopy to linear transformation of the ball $B_{u}$ (left: $B_{1}$, right: $B_{2}$ ) without touching $M^{+}$during this deformation. Moreover, the homotopy keeps the image of exit set $N^{-}$away from $|M|$ in a topologically nontrivial manner; i.e., the image of the exit set $N^{-}$is mapped outside of the unit ball in $h_{M}$ coordinates in projection onto $R^{u_{M}}$.

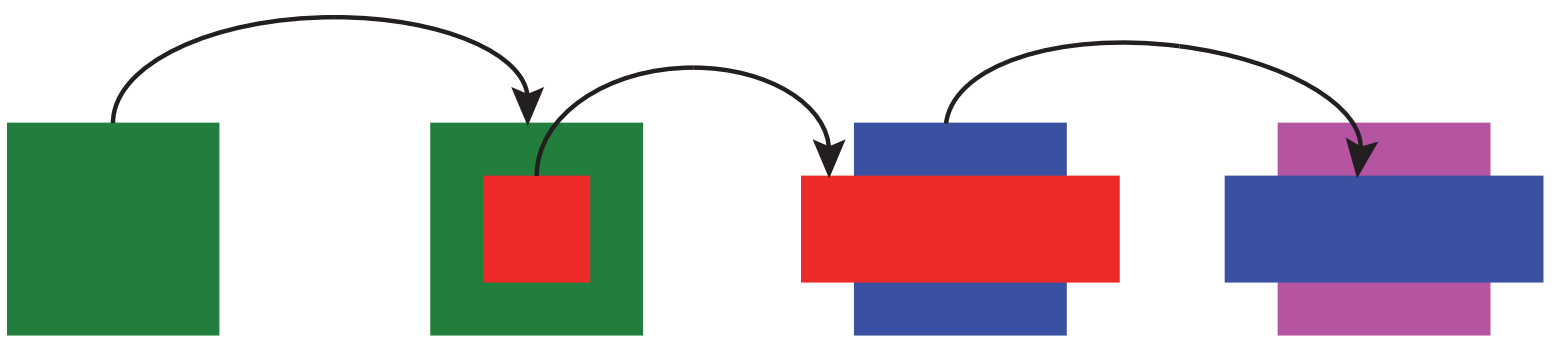

Figure 8. Chain of covering relations with a nonconstant number of unstable directions. Theorem 3.3 guarantees the existence of a shadowing orbit for such a chain.

transit trajectories. The geometry of this case is shown in Figure 8 (see also Figure 18 for its realization into the $4 \mathrm{D}$ Rössler model).

Theorem 3.3. Let $N_{i}=\left(h_{N_{i}}, u_{i}, s_{i}\right), i \in \mathbb{Z}$, be a sequence of $h$-sets such that $u_{i}$ and $s_{i}$ are bounded. Let $f_{i}:\left|N_{i}\right| \rightarrow \mathbb{R}^{n_{i+1}}, i \in \mathbb{Z}$, be continuous maps, where $n_{i}=u_{i}+s_{i}$. If $N_{i} \stackrel{f_{i}}{\Longrightarrow} N_{i+1}$ for $i \in \mathbb{Z}$, then there exists a sequence $\left\{z_{i}\right\}_{i \in \mathbb{Z}}$ such that for $i \in \mathbb{Z}$ there holds that

$$
\begin{aligned}
z_{i} & \in\left|N_{i}\right|, \\
z_{i+1} & =f_{i}\left(z_{i}\right) .
\end{aligned}
$$

Proof. The sequences $\left\{u_{i}, s_{i}\right\}_{i \in \mathbb{Z}}$ are bounded, and so we can set $u=1+\max _{i \in \mathbb{Z}} u_{i}$, $s=1+\max _{i \in \mathbb{Z}} s_{i}$, and $n=u+s$. The number 1 is added just to avoid zero-dimensional cases. We define an $h$-set $M=(\mathrm{Id}, u, s)$, a sequence of permutations (block transpositions)

$$
\sigma_{i}\left(x_{1}, \ldots, x_{n}\right)=(x_{1}, \ldots, x_{u_{i}}, \overbrace{x_{u+1}, \ldots, x_{u+s_{i}}}, \overbrace{x_{u_{i}+1} \ldots, x_{u}}, x_{u+s_{i}+1}, \ldots, x_{n}),
$$

and a sequence of mappings $g_{i}:|M| \rightarrow \mathbb{R}^{n}$ given by

$$
\sigma_{i+1} g_{i}\left[\begin{array}{c}
x_{1} \\
\vdots \\
x_{n}
\end{array}\right]=\left[\begin{array}{c}
\left(h_{N_{i+1}}^{-1} \circ f_{i} \circ h_{N_{i}}\right)\left(x_{1}, \ldots, x_{u_{i}}, x_{u+1}, \ldots, x_{u+s_{i}}\right) \\
2\left(x_{u_{i+1}+1}, \ldots, x_{u}\right) \\
0
\end{array}\right]
$$




$$
\in \mathbb{R}^{n_{i+1}} \times \mathbb{R}^{u-u_{i+1}} \times \mathbb{R}^{s-s_{i+1}} \cong \mathbb{R}^{n}
$$

Note that the main dynamics is given by the mappings $f_{i}$ and we have added some artificial expansion and contraction just to have a constant number of unstable and stable directions.

First, we will show that for $i \in \mathbb{Z}$ there holds that $M \stackrel{g_{i}}{\Longrightarrow} M$. To this end, we define a homotopy required in the definition of covering relation. Let $H_{i}:[0,1] \times B_{u_{i+1}} \times B_{n_{i}-u_{i+1}} \rightarrow$ $\mathbb{R}^{n_{i+1}}$ be the homotopy from the covering relation $N_{i} \stackrel{f_{i}}{\Longrightarrow} N_{i+1}$, and let $L_{i}: \mathbb{R}^{u_{i+1}} \rightarrow \mathbb{R}^{u_{i+1}}$ be the corresponding linear mapping for this covering relation. We define

$$
\widetilde{H}_{i}:[0,1] \times B_{n} \ni\left[\begin{array}{c}
t \\
x_{1} \\
\vdots \\
x_{n}
\end{array}\right] \rightarrow \sigma_{i+1}^{-1}\left[\begin{array}{c}
H_{i}\left(t, x_{1}, \ldots, x_{u_{i}}, x_{u+1}, \ldots, x_{u+s_{i}}\right) \\
2\left(x_{u_{i+1}+1}, \ldots, x_{u}\right) \\
0
\end{array}\right] \in \mathbb{R}^{n}
$$

Since $H_{i}(0, \cdot)=h_{N_{i+1}}^{-1} \circ f_{i} \circ h_{N_{i}}$, we have $\widetilde{H}_{i}(0, \cdot)=g_{i}$ and (3) is satisfied for $\widetilde{H}_{i}$. We also have

$$
\widetilde{H}_{i}\left[\begin{array}{c}
1 \\
x_{1} \\
\vdots \\
x_{n}
\end{array}\right]=\sigma_{i+1}^{-1}\left[\begin{array}{c}
L_{i}\left(x_{1}, \ldots, x_{u_{i+1}}\right) \\
0 \\
2\left(x_{u_{i+1}+1}, \ldots, x_{u}\right) \\
0
\end{array}\right]=\left[\begin{array}{c}
L_{i}\left(x_{1}, \ldots, x_{u_{i+1}}\right) \\
2\left(x_{u_{i+1}+1}, \ldots, x_{u}\right) \\
0 \\
0
\end{array}\right] .
$$

Hence, we have shown that there is a linear mapping $\widetilde{L}_{i}: \mathbb{R}^{u} \rightarrow \mathbb{R}^{u}$ such that

$$
\widetilde{H}_{i}\left(1, x_{1}, \ldots, x_{n}\right)=\left(\widetilde{L}_{i}\left(x_{1}, \ldots, x_{u}\right), 0\right) .
$$

It is easy to check that the spectrum of $\widetilde{L}_{i}$ is equal to the union of the spectrum of $L_{i}$ and $\{2\}$, and therefore (4) is satisfied for $\widetilde{H}_{i}$.

We will check condition (5). Let us fix $t \in[0,1]$ and $\left(x_{1}, \ldots, x_{n}\right) \in \partial B_{u} \times B_{s}$. Thus $\left|x_{j}\right|=1$ for some $j \in\{1, \ldots, u\}$. If $j \leq u_{i+1}$, then from (5) we have

$$
H_{i}\left(t, x_{1}, \ldots, x_{u_{i}}, x_{u+1}, \ldots, x_{u+s_{i}}\right) \notin B_{n_{i+1}},
$$

and thus $\widetilde{H}_{i}\left(t, x_{1}, \ldots, x_{n}\right) \notin B_{n}$. If $j>u_{i+1}$, then the absolute value of the $j$ th component of $\widetilde{H}_{i}\left(t, x_{1}, \ldots, x_{n}\right)$ is equal to 2 , and thus $\widetilde{H}_{i}\left(t, x_{1}, \ldots, x_{n}\right) \notin B_{n}$. This proves that $(5)$ holds for $\widetilde{H}_{i}$.

Assume now that $\widetilde{H}_{i}\left(t, x_{1}, \ldots, x_{n}\right) \in B_{u} \times \partial B_{s}$. This is possible only if

$$
H_{i}\left(t, x_{1}, \ldots, x_{u_{i}}, x_{u+1}, \ldots, x_{u+s_{i}}\right) \in B_{u_{i+1}} \times \partial B_{s_{i+1}},
$$

which contradicts assumption (6) on $H_{i}$. Therefore, (6) holds also for $\widetilde{H}_{i}$.

Since $M \stackrel{g_{i}}{\Longrightarrow} M$ for all $i \in \mathbb{Z}$, from [23, Corollary 8] there exists a sequence $\left\{y_{i}\right\}_{i \in \mathbb{Z}}$ such that $y_{i} \in|M|$ and $g_{i}\left(y_{i}\right)=y_{i+1}$ hold true for $i \in \mathbb{Z}$. Put

$$
z_{i}=\left(h_{N_{i}} \circ \pi_{i} \circ \sigma_{i}\right)\left(y_{i}\right)
$$


where $\pi_{i}$ is a canonical projection onto first $n_{i}$ coordinates. Clearly, $z_{i} \in\left|N_{i}\right|$ and, by the definition of $g_{i}$, we have $f_{i}\left(z_{i}\right)=z_{i+1}$, which completes the proof.

For self-consistency of this paper we will shortly recall the method of two-point cone conditions introduced in [28] and extended to the periodic case in [55].

Definition 3.4. A quadruple $N=\left(h_{N}, u, s, Q\right)$ is called an $h$-set with cones if

- $u, s$ are natural numbers,

- $h_{N}$ is a homeomorphism of $\mathbb{R}^{u+s}$, and

- $Q(x, y)=\alpha(x)-\beta(y)$ is a quadratic form on $\mathbb{R}^{u} \times \mathbb{R}^{s}$ such that $\alpha$ and $\beta$ are positive definite.

Definition 3.5. Let $N=\left(h_{N}, u, s, Q_{N}\right)$ and $M=\left(h_{M}, u, s, Q_{M}\right)$ be h-sets with cones, and let $f:|N| \rightarrow \mathbb{R}^{u+s}$ be a continuous map. Put $f_{N, M}=h_{M}^{-1} \circ f \circ h_{N}$. We say that $N f$-covers $M$ with cones if $N \stackrel{f}{\Longrightarrow} M$ and for $u, v \in B_{n}$ there holds that

$$
Q_{M}\left(f_{N, M}(u)-f_{N, M}(v)\right) \geq Q_{N}(u-v) .
$$

The geometry of Definition 3.5 of two-point cone conditions is shown in Figure 9.

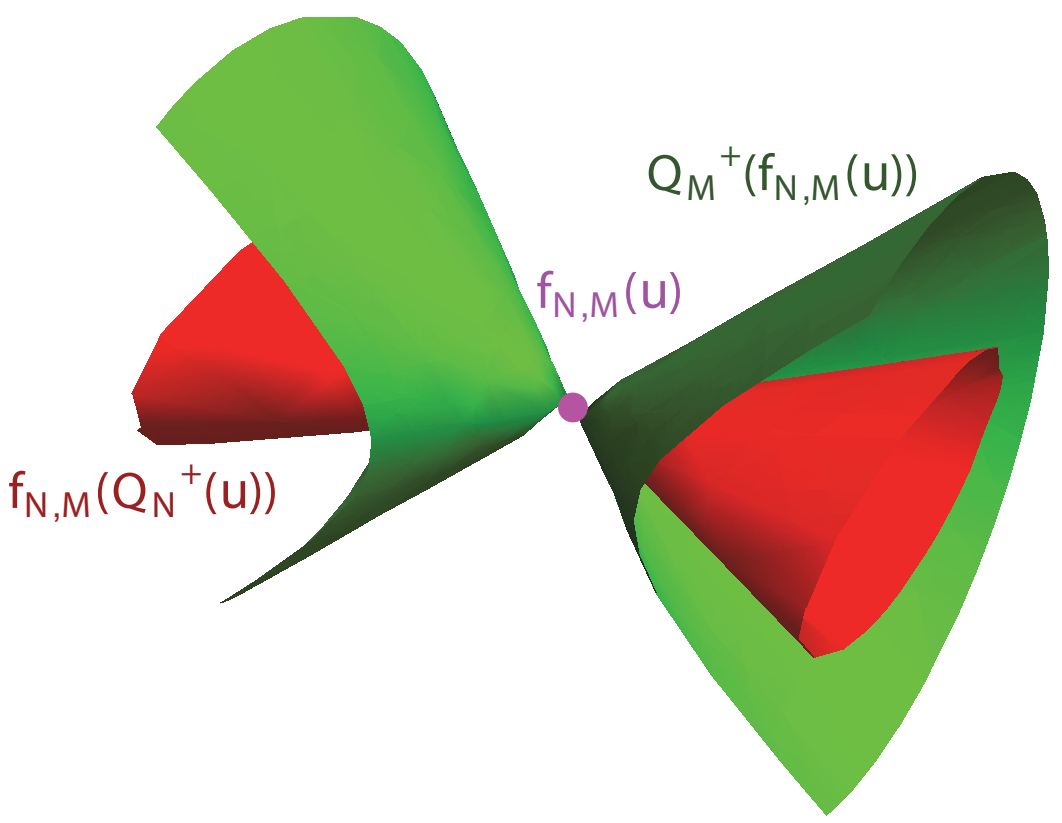

Figure 9. Geometry of two-point cone conditions from Definition 3.5. The positive cone attached to any point $u \in B_{n}$ is mapped into a positive cone attached to $f_{N, M}(u)$.

Remark 3.6. If $f:|N| \rightarrow \mathbb{R}^{u+s}$ is smooth and the coordinate systems $h_{N}$ and $h_{M}$ are diffeomorphisms, then the positive definiteness of the interval matrix

$$
D f_{N, M}^{T}(u) Q_{M} D f_{N, M}(u)-Q_{N}
$$


for all $u \in B_{n}$ implies that for $u, v \in B_{n}$ the condition

$$
Q_{M}\left(f_{N, M}(u)-f_{N, M}(v)\right) \geq Q_{N}(u-v)
$$

is satisfied. The positive definiteness of an interval matrix is an open condition and thus suitable for rigorous numerics.

The next theorem gives us a tool for proving convergence of a (backward or forward) trajectory to a periodic point.

Theorem 3.7 (see [62, Thms. 10,12]). Let $N=\left(h_{N}, u, s, Q_{N}\right)$ be an h-set with cones, and let $f:|N| \rightarrow \mathbb{R}^{u+s}$ be a continuous map. If $N f$-covers $N$ with cones, then the set $|N|$ contains exactly one fixed point $x_{*} \in|N|$ for $f$.

Moreover, if $f$ is a diffeomorphism on $|N|$ and $h_{N}$ is smooth, then local unstable and stable manifolds of $x_{*}$ defined as

$$
\begin{aligned}
& W_{N}^{u}\left(x_{*}, f\right):=\left\{x \in|N|: \forall k>0 f^{-k}(x) \in|N| \text { and } \lim _{k \rightarrow \infty} f^{-k}(x)=x_{*}\right\}, \\
& W_{N}^{s}\left(x_{*}, f\right):=\left\{x \in|N|: \forall k>0 f^{k}(x) \in|N| \text { and } \lim _{k \rightarrow \infty} f^{k}(x)=x_{*}\right\}
\end{aligned}
$$

are given by

$$
\begin{aligned}
& W_{N}^{u}\left(x_{*}, f\right)=\left\{h_{N}\left(g_{u}(x), x\right): x \in B_{u}\right\}, \\
& W_{N}^{s}\left(x_{*}, f\right)=\left\{h_{N}\left(y, g_{s}(y)\right): y \in B_{s}\right\},
\end{aligned}
$$

where $g_{u}: B_{u} \rightarrow B_{s}$ and $g_{s}: B_{s} \rightarrow B_{u}$ are Lipschitz functions. In particular, they are of dimensions $u$ and $s$, respectively.

Now we have all the mathematical machinery to come back to our problem, the 4D Rössler system.

4. Rigorous results about the 4D Rössler system. The CAP study of the 4D Rössler system was initiated in [55]. In that paper the existence of chaotic dynamics with two expanding directions (hyperchaos) for the classical parameter values $a=0.25, b=3, c=0.5$, and $d=0.05$ was proved. These parameter values are located out of the biparametric plot of Figure 3, but the point is placed on the upper-left area, where a robust hyperchaotic behavior is observed.

In this section we focus on the parameter values for which coexistence of various types of dynamics is observed by giving details of the CAP of the coexistence of chaos and hyperchaos in the 4D Rössler system. Moreover, we will also show that there is a dynamical link between hyperchaotic and chaotic invariant sets, and this gives rise to a possible route of the smooth transition from chaotic to hyperchaotic behavior.

Consider again the 4D Rössler system (2) with the parameter values $a=0.27857, b=3$, $c=0.3, d=0.05$. In Figure 10 we plot the hyperchaotic saddle and the chaotic attractor that generate the different dynamics for these parameter values (see Figures 3, 4, and 6). Note that there is a large hyperchaotic invariant set with a saddle dynamics, which means that the dynamics of the surrounding orbits spend some time close to it, but finally they go to the chaotic attractor of a smaller size. We remark the differences with the case studied in [55], 


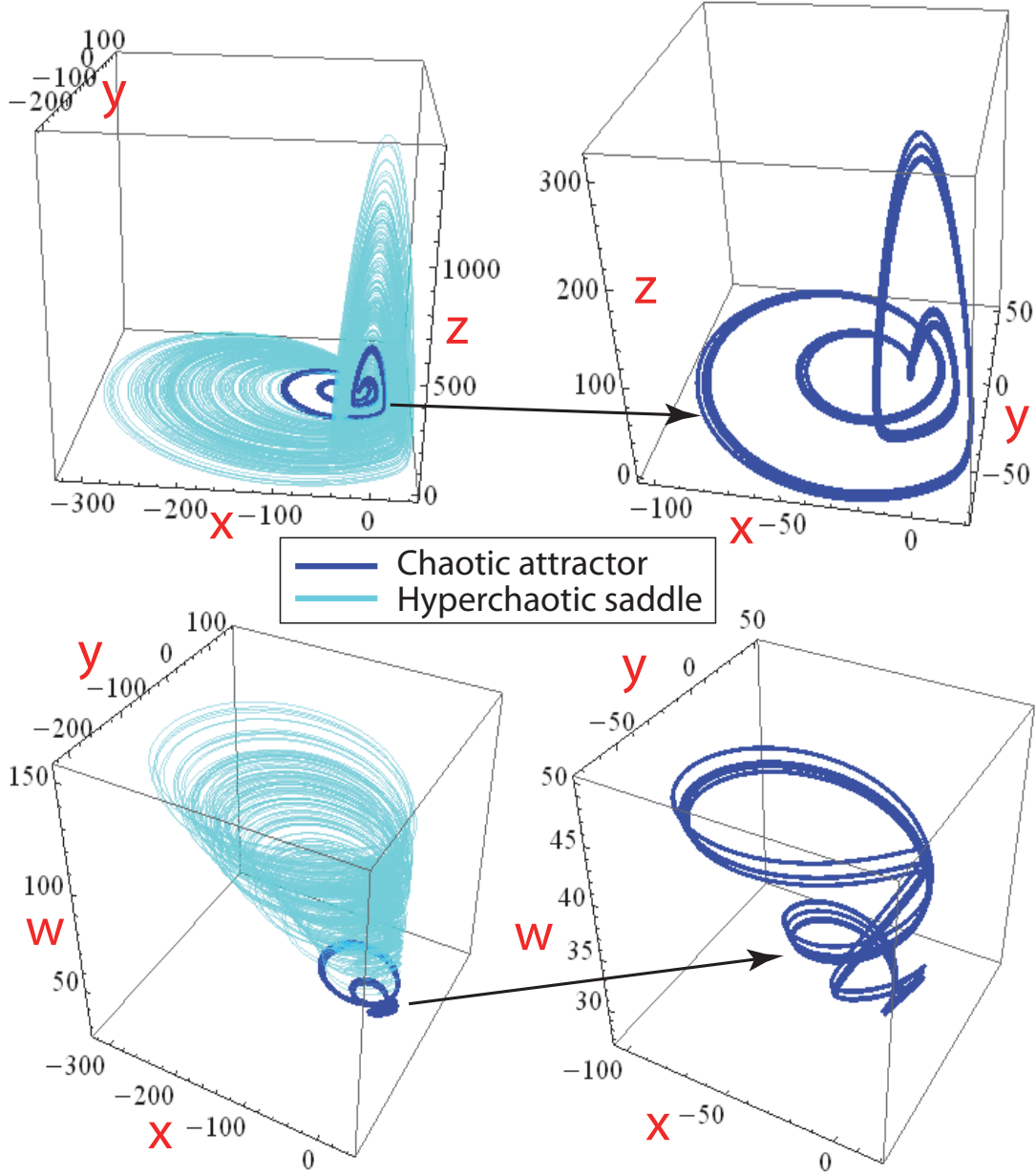

Figure 10. Two projections of the hyperchaotic saddle and of the chaotic attractor of the $4 D$ Rössler system at the parameter values $a=0.27857, b=3, c=0.3, d=0.05$.

where the attractor is a hyperchaotic attractor (very similar to case A plotted on the bottom of Figure 4).

Let

$$
\Pi=\left\{(x, 0, z, w) \in \mathbb{R}^{4}, \dot{y}=x+w<0\right\}
$$

be a complete Poincaré section [18], and let $P: \Pi \rightarrow \Pi$ be the associated Poincaré map. In Figure 11 we show $(x, y)$ projection of a typical trajectory of (2) and the Poincaré section. Due to its definition, to describe points on section $\Pi$ we will use $(x, z, w)$ coordinates only.

The first question to prove is the existence of a trapping region $\mathcal{B}$ of the Poincaré map $P$, that is, a compact set $\mathcal{B}$ such that $P(\mathcal{B}) \subset \operatorname{int}(\mathcal{B})$. This result generates an enclosure of some invariant set, which is expected to be an attractor of the system.

Theorem 4.1. There exists an explicit given trapping region $\mathcal{B}$ for $P$.

Proof. The CAP uses [56, Algorithm 1], which allows one, if the algorithm stops without Failure, to compute the combinatorial representation of a map restricted to some positive 


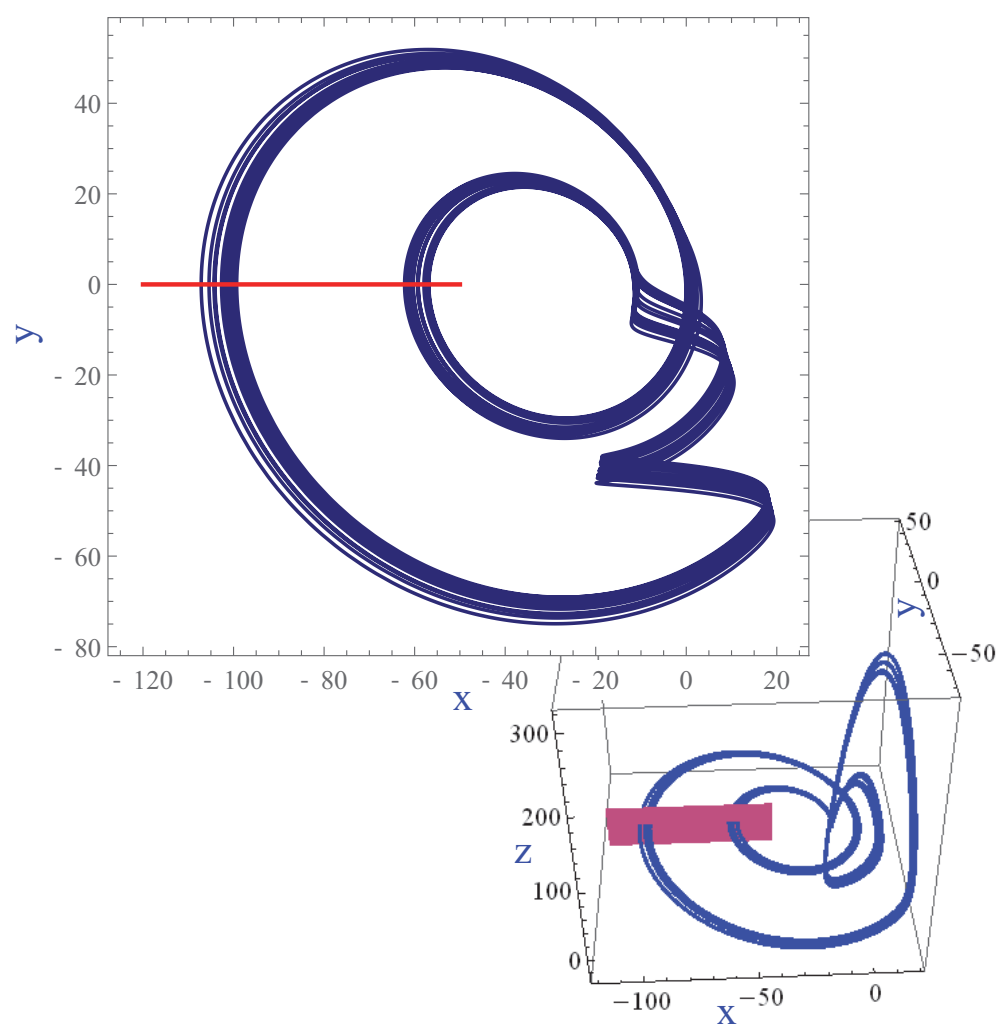

Figure 11. Chaotic attractor and Poincaré section $\Pi$.

invariant set, which is expected to be an attractor. To that goal, and in a few words, the algorithm uses a cover $\mathcal{X}$ of the numerically observed attracting domain. Then, we choose an initial box that contains points from the attractor. We have taken a box $V$ that contains an approximate unstable periodic point for $P$ in the attracting region we have detected numerically:

$$
(-104.42324539012806,0.028730815749171541,44.678254866134068) \in V \text {. }
$$

The algorithm encloses its forward trajectory using the sets from the cover $\mathcal{X}$ as long as the trajectory does not leave $\operatorname{supp}(\mathcal{X})$. Since the cover $\mathcal{X}$ is finite by its definition, after a finite number of steps there are no new sets in the cover of the trajectory of $V$, and we can stop the procedure. We have run the program which implements a parallel version of [56, Algorithm 1] on a computer with $64 \operatorname{Intel}(\mathrm{R}) \mathrm{Xeon}(\mathrm{R}) \mathrm{CPU}$ E7-8837 2.67GHz cores. The program stopped after 162 minutes and returned a positive invariant set $\mathcal{B}$ for $P$ which is the union of 22683543 boxes of size $\left(2^{-10}, 2^{-10}, 2^{-12}\right)$.

Three projections of the positive invariant set $\mathcal{B}$, found by the algorithm, onto each pair of coordinates are shown in Figure 12.

Now, we are interested in studying the dynamics inside the trapping region. Note that we have obtained a trapping region that encloses the numerically detected attractor, but, 

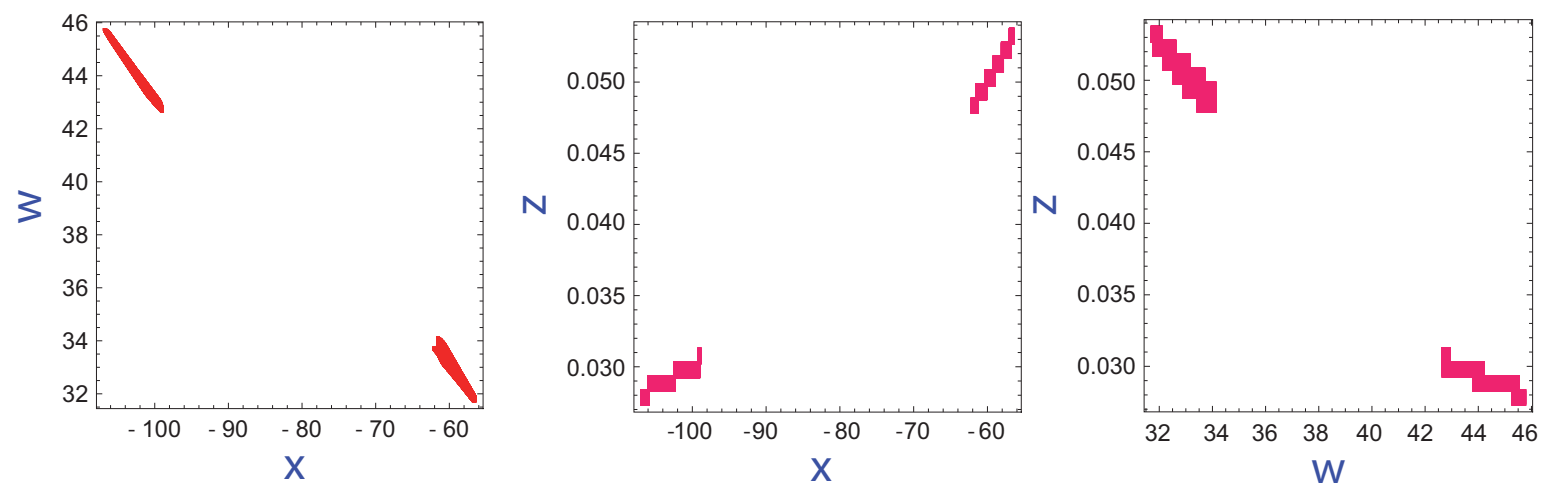

Figure 12. Three projections of the trapping region $\mathcal{B}$ for Poincaré map $P$ returned by [56, Algorithm 1]; see Theorem 4.1.

also from the numerics, the system has a large hyperchaotic saddle invariant set that goes outside the trapping region. That is, although our proof studies the dynamics in $\mathcal{B}$, similar hyperchaotic structures, such as those in $\mathcal{B}$, are expected to be outside creating all together this saddle invariant set.

By $\mathcal{A}$ we denote the maximal invariant set for $P$ in $\mathcal{B}$, i.e.,

$$
\mathcal{A}=\operatorname{inv}(P, \mathcal{B})=\bigcap_{n>0} P^{n}(\mathcal{B})
$$

We will show that the dynamics of $P$ on $\mathcal{A}$ admits different types of chaos. Moreover, we will show that there are heteroclinic orbits linking a hyperchaotic horseshoe with two different chaotic horseshoes.

Using the same approach as [56, section 3.3] we have found many approximate unstable periodic points (UPPs) for $P$ (UPOs of the flow). An extensive numerical study yields finding heteroclinic connections between some of these periodic points. We will show that these periodic orbits and heteroclinic connections indeed exist, giving rise to various types of chaotic dynamics.

From the different UPPs, we have selected the set $\left\{p_{1}^{8}, p_{2}^{8}, p_{3}^{8}, p_{4}^{16}, q_{1}^{12}, q_{2}^{14}\right\}$ with the initial conditions in Poincaré section $\Pi$ :

$$
\begin{aligned}
p_{1}^{8} & =(-104.32937253702462,0.028756669726685443,44.645081351998819), \\
p_{2}^{8} & =(-104.26664163365506,0.028773972266421831,44.640115482927115), \\
p_{3}^{8} & =(-104.42324539012806,0.028730815749171541,44.678254866134068), \\
p_{4}^{16} & =(-104.39575243552828,0.028738382959034744,44.666264617071981), \\
q_{1}^{12} & =(-103.69667754570543,0.028932144798038389,44.407870627484129), \\
q_{2}^{14} & =(-103.37098255164607,0.029023312473829044,44.284349486019579) .
\end{aligned}
$$

Projections onto the $(x, y)$ plane of periodic trajectories corresponding to the points listed in (7) are shown in Figure 13. The points $p_{i}^{j}$ are approximate periodic points for $P$ of period $j$ with a one-dimensional unstable manifold. The points $q_{i}^{j}$ are approximate periodic points of period $j$ with a two-dimensional unstable manifold. The following theorem says that the above points are good numerical approximations of unstable and hyperbolic periodic points for $P$. 

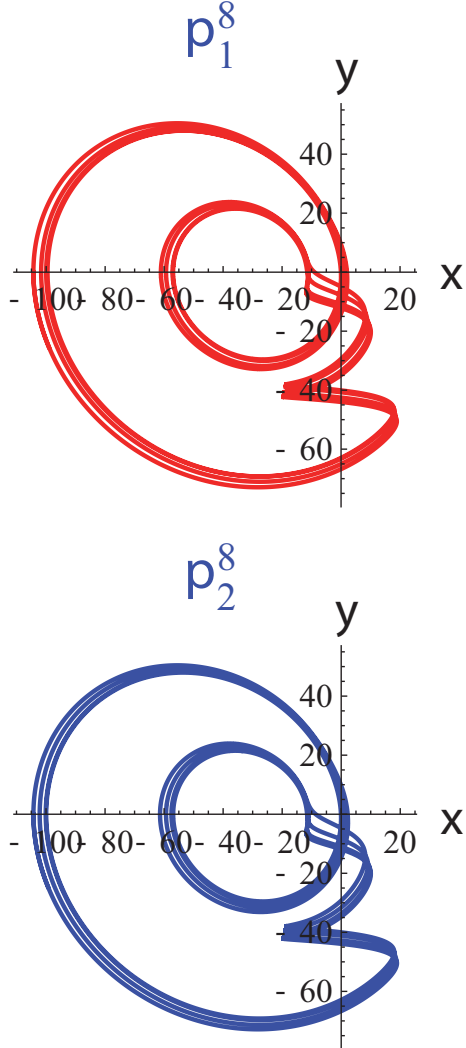
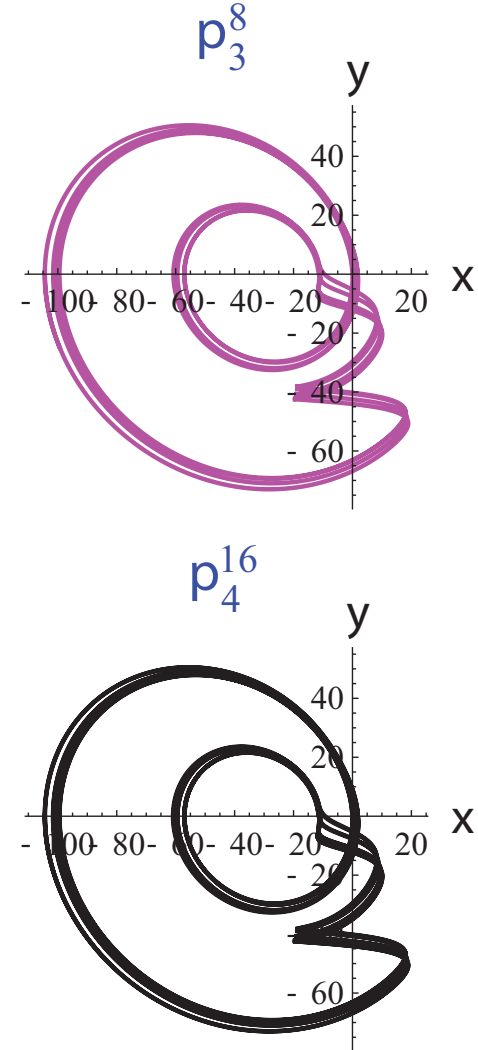
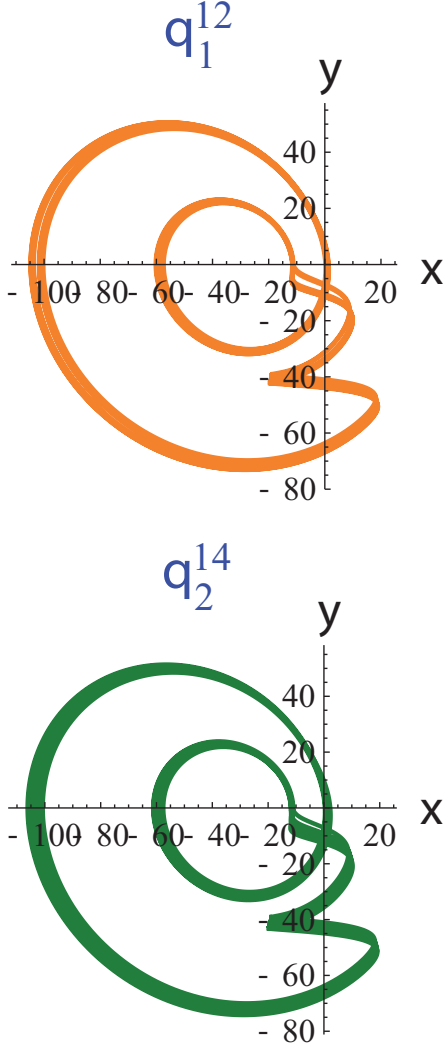

Figure 13. Unstable periodic orbits resulting from Theorem 4.2.

Theorem 4.2. For each $u^{j} \in\left\{p_{1}^{8}, p_{2}^{8}, p_{3}^{8}, p_{4}^{16}, q_{1}^{12}, q_{2}^{14}\right\}$ there is a unique hyperbolic periodic orbit $v$ for $P$ of the principal period $j$ in the ball $B\left(u^{j}, 10^{-8}\right)$ in the maximum norm.

Moreover, the resulting periodic points close to $p_{i}^{j}$ have a one-dimensional unstable invariant manifold and those corresponding to $q_{i}^{j}$ have a two-dimensional unstable invariant manifold.

Proof. For $j>1$ we define $F_{j}: \Pi^{j} \rightarrow \mathbb{R}^{3 j}$ by

$$
F_{j}\left(v_{1}, v_{2}, \ldots, v_{j}\right)=\left(v_{1}-P\left(v_{j}\right), v_{2}-P\left(v_{1}\right), \ldots, v_{j}-P\left(v_{j-1}\right)\right) .
$$

Clearly, solutions to $F_{j}\left(v_{1}, v_{2}, \ldots, v_{j}\right)=0$ correspond to $j$-periodic orbits for $P$, provided that $v_{i} \neq v_{c}$ for $i \neq c$.

Let us fix $u^{j} \in\left\{p_{1}^{8}, p_{2}^{8}, p_{3}^{8}, p_{4}^{16}, q_{1}^{12}, q_{2}^{14}\right\}$ and put

$$
z_{0}=\left(v_{1}, v_{2}, \ldots, v_{j}\right)=\left(u^{j}, \hat{P}\left(u^{j}\right), \hat{P}^{2}\left(u^{j}\right), \ldots, \hat{P}^{j-1}\left(u^{j}\right)\right),
$$

where by $\hat{P}(u)$ we denote an approximate value of $P(u)$ obtained by a suitable nonrigorous numerical method.

Let $Z=B\left(z_{0}, 10^{-8}\right)$ be the ball centered at $z_{0}$ in the maximum norm. Using rigorous solvers for ODEs and variational equations [50, 61] from the CAPD library [15] we compute 
the interval Newton operator [37]

$$
N\left(F_{j}, Z, z_{0}\right)=\left(N_{1}, \ldots, N_{j}\right)=z_{0}-\left[D F_{j}(Z)\right]_{I}^{-1} \cdot F_{j}\left(z_{0}\right),
$$

and we obtain that $N\left(F_{j}, Z, z_{0}\right) \subset \operatorname{int}(Z)$. This proves that $F_{j}$ has unique zero $\left(v_{1}, \ldots, v_{j}\right)$ in $Z$. Moreover, this zero belongs to $N\left(F_{j}, Z, z_{0}\right)$, which in most cases had diameter less than $10^{-9}$. From these estimations we could conclude that $v_{1}$ is a periodic point for $P$ with principal period $j$. Therefore, we have already rigorously proved the existence and uniqueness of the corresponding periodic orbit in a small ball centered at $u^{j}$.

In what follows we will explain how we verify the stability type of a periodic orbit. Let $[M]$ be a complex interval matrix, and let $\lambda^{0} \in \mathbb{C}$ be an approximate eigenvalue of the center of $[M]$ with corresponding approximate eigenvector $w^{0}=\left(w_{1}^{0}, \ldots, w_{n}^{0}\right)^{T}$. After reordering the coordinates we may assume that $w_{1}^{0} \neq 0$. In order to find a rigorous bound for the set of eigenvalues of $[M]$, we can apply the interval Newton operator to the nonlinear map

$$
G\left(\lambda, w_{2}, \ldots, w_{n}\right)=([M]-\lambda \mathrm{Id})\left(w_{1}^{0}, w_{2}, \ldots, w_{n}\right)^{T}
$$

for every approximate zero $\left(\lambda^{0}, w_{2}^{0}, \ldots, w_{n}^{0}\right)$. For this purpose we choose an interval vector $\widetilde{W}$ centered at $\bar{w}^{0}=\left(\lambda^{0}, w_{2}^{0}, \ldots, w_{n}^{0}\right)$, and then we check the inclusion

$$
N=\bar{w}^{0}-[D G(\widetilde{W})]_{I}^{-1} G\left(\bar{w}^{0}\right) \subset \operatorname{int} \widetilde{W} .
$$

If we succeed, then the first coefficient $N_{1}$ is a rigorous bound for one of the eigenvalues of $[M]$ and $\left(w_{1}^{0}, N_{2}, \ldots, N_{n}\right)$ is an interval vector that encloses the corresponding eigenvector of $[M]$. Using rigorous solvers for the variational equations for ODEs from the CAPD library [15] we computed a rigorous bound for $D P^{j}\left(v_{1}\right) \in D P^{j}\left(N_{1}\right)$. Then applying the interval Newton method as described above we checked the hyperbolicity type of the periodic point $v_{1}$ by analysis of the spectrum of the interval matrix $\left[D P^{j}\left(N_{1}\right)\right]$. The actual bounds for eigenvalues

\begin{tabular}{|c|c|c|c|c|}
\hline Orbit & $\lambda_{1}$ & $\lambda_{2}$ & $\lambda_{3}$ & Flow time \\
\hline$p_{1}^{8}$ & $2.7_{4}^{6}$ & $-0.16_{4}^{6}$ & {$[-1,1] \cdot 10^{-11}$} & $56.585531937_{00}^{57}$ \\
\hline$p_{2}^{8}$ & $-3.9_{89}^{91}$ & $-0.54_{4}^{7}$ & {$[-5,5] \cdot 10^{-12}$} & $56.59832252_{61}^{73}$ \\
\hline$p_{3}^{8}$ & -1.91 & $0.36_{4}^{6}$ & {$[-1,1] \cdot 10^{-11}$} & $56.593931_{89}^{91}$ \\
\hline$p_{4}^{16}$ & {$[-3.30,-2.96]$} & \multicolumn{2}{|c|}{$[-0.05,0.26]+[-0.13,0.13] i$} & $113.1853_{59}^{61}$ \\
\hline$q_{1}^{12}$ & $-22 \cdot 31$ & $-3 .{ }_{06}^{14}$ & {$[-8,8] \cdot 10^{-11}$} & $85.0883812_{29}^{36}$ \\
\hline$q_{2}^{14}$ & $-4 \cdot{ }_{39}^{47}+5 \cdot \cdot_{36}^{48} \mathrm{i}$ & $-4.47-5 \cdot{ }_{36}^{48} \mathrm{i}$ & {$[-5,5] \cdot 10^{-11}$} & $99.2973738_{34}^{53}$ \\
\hline
\end{tabular}
$\left\{\lambda_{1}, \lambda_{2}, \lambda_{3}\right\}$ are the following:

Note that the intervals that enclose the eigenvalues are clearly inside or outside the unit circle. This shows that these six periodic points are hyperbolic. From the above data it is also clear that the points $p_{i}^{j}$ have a one-dimensional unstable manifold and the points $q_{i}^{j}$ have a two-dimensional unstable manifold.

Remark 4.3. Nonrigorous simulation strongly indicates that all eigenvalues of $\operatorname{DP}^{16}\left(p_{4}^{16}\right)$ are real and distinct. However, the bounds for eigenvalues obtained in rigorous computation 
are not sharp enough to conclude whether $\lambda_{2}$ and $\lambda_{3}$ are two real or complex conjugate eigenvalues. Even so, we can conclude from these estimates that the orbit $p_{4}^{16}$ is hyperbolic with one expanding and two contracting directions.

Remark 4.4. In the macroscopic scale the periodic orbits resulting from Theorem 4.2 look similar (see Figure 13), but they have different types of stability. There are other periodic orbits with a two-dimensional unstable manifold even closer to chaotic region, for instance,

$$
\begin{aligned}
& q_{3}^{8}=(-104.33185446775647,0.028755984103875101,44.703879687853039), \\
& q_{4}^{12}=(-104.62637739731338,0.028675025582387843,44.887495900175296), \\
& q_{5}^{16}=(-104.86937675403779,0.028608575265772675,44.919900861034812) .
\end{aligned}
$$

The point $q_{3}^{8}$ is quite close to $p_{1}^{8}$. The point $q_{5}^{16}$ has a pair of complex eigenvalues out of the unit disc. It turned out, however, that it was easier to find hyperchaotic horseshoe and heteroclinic transit from hyperchaos to chaos when using the pair $q_{1}$ and $q_{2}$. The details will be explained in Theorem 4.5 .

The next theorem is the main rigorous numerical result in this section. It shows the coexistence of chaotic and hyperchaotic invariant sets and the existence of dynamical links between these sets.

Theorem 4.5. Denote by $p_{1}, p_{2}, p_{3}, p_{4}, q_{1}, q_{2}$ the periodic points for $P$ resulting from Theorem 4.2. Then for every bi-infinite path $\left\{v_{j}\right\}_{j \in \mathbb{Z}} \in\left\{p_{1}, p_{2}, p_{3}, p_{4}, q_{1}, q_{2}\right\}^{\mathbb{Z}}$ on the graph shown in Figure 14 there exists a sequence $\left\{y_{j}\right\}_{j \in \mathbb{Z}}$ such that for $j \in \mathbb{Z}$ there hold that

$$
\begin{aligned}
\left\|y_{j}-v_{j}\right\|_{1} & \leq 10^{-3}, \\
P^{n_{j}}\left(y_{j}\right) & =y_{j+1},
\end{aligned}
$$

where $n_{j}$ is the weight of the edge $\left(v_{j}, v_{j+1}\right)$.

Moreover,

- if $\left\{v_{j}\right\}_{j \in \mathbb{Z}}$ is periodic, then the sequence $\left\{y_{j}\right\}_{j \in \mathbb{Z}}$ can be chosen to be a periodic point for $P$;

- if $\left\{v_{j}\right\}_{j \in \mathbb{Z}}$ is nonconstant and of the form

$$
\left(v_{0}\right)^{\mathbb{N}}\left(v_{0}, \ldots, v_{n}\right)\left(v_{n}\right)^{\mathbb{N}},
$$

then the sequence $\left\{y_{j}\right\}_{j \in \mathbb{Z}}$ can be chosen as a heteroclinic orbit between periodic points $v_{0}$ and $v_{n}$. If $v_{0}=v_{n}$, then this is a homoclinic orbit.

The main elements of this theorem are shown in Figure 14, where the graph of symbolic dynamics among the sets $p_{1}, p_{2}, p_{3}, p_{4}, q_{1}, q_{2}$ of periodic orbits of the $4 \mathrm{D}$ Rössler system is shown. The weights of edges stand for the number of iterations necessary to transport some points from the vicinity of one periodic point (beginning of arrow) to the vicinity of the second periodic point (end of arrow), as obtained in the proof of the theorem (see the next section).

We would like to emphasize some consequences of Theorem 4.5 before we give details of its CAP.

Corollary 4.6. The maximal invariant set $\mathcal{A}=\operatorname{inv}(P, \mathcal{B})$ contains three invariant subsets $S_{1}, S_{2}, S_{3}$ (see Figure 1) on which the dynamics of $P$ is semiconjugated to the Bernoulli shift on two symbols. These horseshoes are built on three pairs of unstable periodic orbits and heteroclinic connections in both directions between them: 


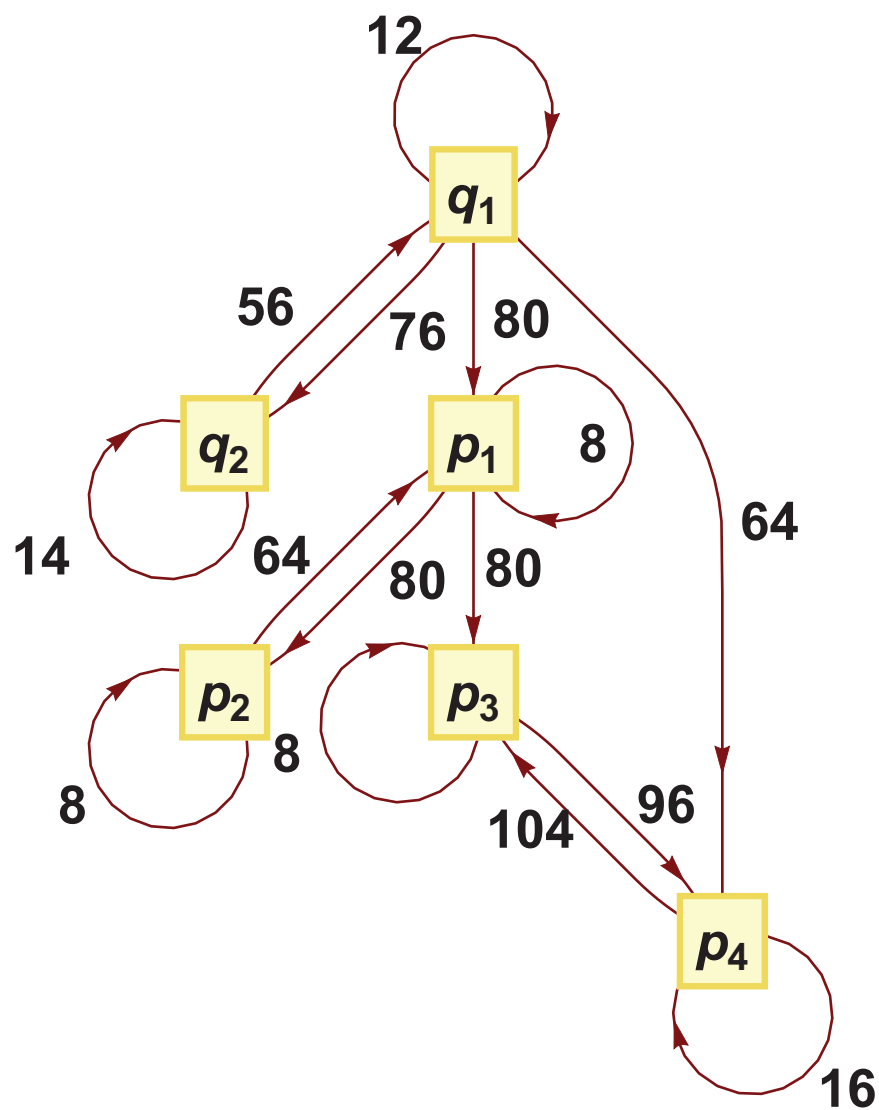

Figure 14. Graph of symbolic dynamics for the $4 D$ Rössler system (2).

- $S_{1}$ is hyperchaotic set built on $\left(q_{1}, q_{2}\right)$;

- $S_{2}, S_{3}$ are chaotic sets built on $\left(p_{1}, p_{2}\right)$ and $\left(p_{3}, p_{4}\right)$, respectively.

Corollary 4.7. The dynamics in $\mathcal{B}$ admits gradient-like structure. The trapping region contains hyperchaotic invariant set $S_{1}$, which is connected via infinitely many heteroclinic orbits to chaotic invariant sets $S_{2}$ and $S_{3}$; see Figure 1 . This gives rise to long transit dynamics from hyperchaos to chaos and explains noise observed in the numerical computation of Lyapunov exponents.

Remark 4.8. Theorem 4.5 gives a partial description of the dynamics in $\mathcal{A}$ only. There might be other invariant sets, like attracting periodic orbits with very small basins of attraction that are not visible even in extensive and very accurate numerical simulation. Besides, outside the trapping region there are more hyperchaotic sets completing the structure of the hyperchaotic saddle.

Remark 4.9. Although the results are proved for just one set of parameters, we note that all the methods used in CAPs are robust under perturbations. Therefore the same statements hold true for all parameter values from some (unknown) neighborhood of those selected by the authors. We remark that in all the numerical simulations done by the authors we can observe 
the same picture, supporting these statements as a common situation in the system.

5. Proof of Theorem 4.5. In this section we will give some details of the CAP of Theorem 4.5. The proof, although computer-assisted, is not fully automatic, and many parameters were set by hand to make the computations possible to perform in reasonable CPU time. As a consequence, presenting all details would take many pages of highly technical data. Therefore we will present here the main ideas and sample numerical data moving all details to the accompanying supplementary material (M103920_01.zip [local/web 8.49MB]) and the C++ code included in it.

The discussion will be split into two parts: the existence of symbolic dynamics and the existence of homoclinic/heteroclinic orbits.

5.1. Symbolic dynamics. The main topological tool for the proof of the existence of symbolic dynamics is Theorem 3.3. All $h$-sets used in the proof will be defined as cubes in some affine coordinates. Therefore, the homeomorphism $h$ from the definition of an $h$-set will always take the form $x \rightarrow v+A \cdot D \cdot x$, where $v \in \mathbb{R}^{n}, A \in \mathbb{R}^{n^{2}}$ is an isomorphism and $D$ is a diagonal matrix with real and positive coefficients. In this case we will write

$$
N=N\left(h_{N}, u, s\right) \equiv N(\overbrace{v, A, D}, u, s) .
$$

In this representation the support $|N|$ is the parallelogram centered at $v$ and spanned on vectors given by columns (usually normalized) of $A$ scaled by diagonal coefficients of $D$.

First we construct six $h$-sets centered at $v_{i}^{j} \in\left\{p_{1}^{8}, p_{2}^{8}, p_{3}^{8}, p_{4}^{16}, q_{1}^{12}, q_{2}^{14}\right\}$ (see (7)) and spanned on approximate eigenvectors of $D P^{j}\left(v_{i}^{j}\right)$. Let $V_{i}^{j}$ be the $h$-set

$$
V_{i}^{j}=V_{i}^{j}(\overbrace{v_{i}^{j}, A_{i}^{j}, D_{i}^{j}}, u_{i}^{j}, s_{i}^{j}),
$$

where $\left(u_{i}^{j}, s_{i}^{j}\right)=(2,1)$ for $v_{i}^{j} \in\left\{q_{1}^{12}, q_{2}^{14}\right\}$ and $\left(u_{i}^{j}, s_{i}^{j}\right)=(1,2)$ otherwise. The matrices $A_{i}^{j}$ and $D_{i}^{j}$ are the following:

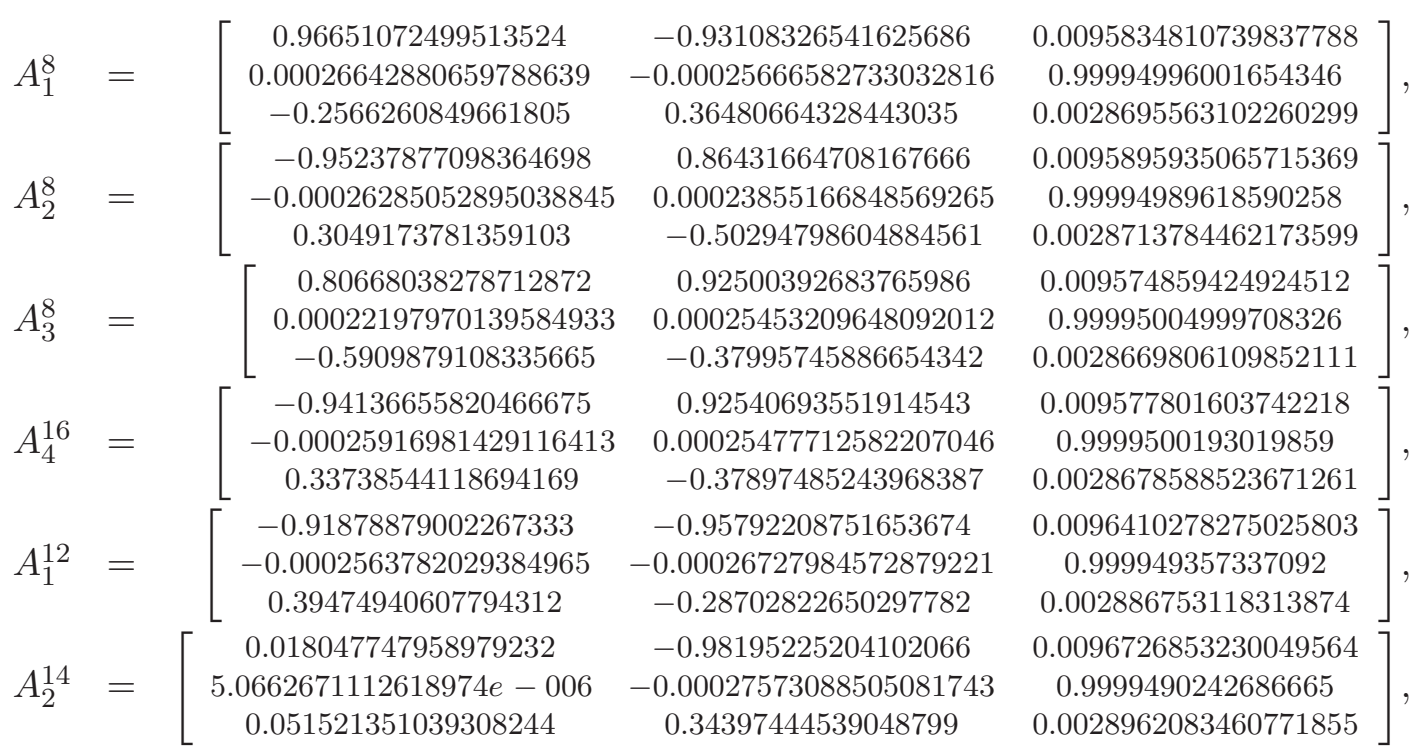


and

$$
\begin{aligned}
D_{1}^{8} & =\operatorname{Diag}\left(10^{-3}, 10^{-4}, 10^{-7}\right), \\
D_{2}^{8} & =\operatorname{Diag}\left(10^{-3}, 6 \cdot 10^{-4}, 10^{-7}\right), \\
D_{3}^{8} & =\operatorname{Diag}\left(10^{-4}, 6 \cdot 10^{-4}, 10^{-7}\right), \\
D_{4}^{16} & =\operatorname{Diag}\left(10^{-3}, 10^{-3}, 10^{-7}\right), \\
D_{1}^{12} & =\operatorname{Diag}\left(10^{-5}, 5 \cdot 10^{-5}, 10^{-6}\right), \\
D_{2}^{14} & =\operatorname{Diag}\left(3.5 \cdot 10^{-4}, 2 \cdot 10^{-4}, 10^{-6}\right) .
\end{aligned}
$$

All but $A_{2}^{14}$ matrices have normalized columns. This matrix corresponds to the periodic point $q_{2}$ which has complex eigenvalues (see (8)), and due to some algorithms for checking the cone conditions it is better to keep them as the real and imaginary parts of the corresponding complex (normalized) eigenvector.

Using the above technical initial data, the following lemma has been verified with computer assistance. This is the first step in the proof of the existence of symbolic dynamics in Theorem 4.5.

Lemma 5.1. For $V_{i}^{j} \in\left\{V_{1}^{8}, V_{2}^{8}, V_{3}^{8}, V_{4}^{16}, V_{1}^{12}, V_{2}^{14}\right\}$ there holds that

$$
V_{i}^{j} \stackrel{P^{j}}{\Longrightarrow} V_{i}^{j}
$$

Moreover, for $x \in\left|V_{i}^{j}\right|$ there holds that

$$
\left\|x-v_{i}^{j}\right\|_{1} \leq 0.97 \cdot 10^{-3},
$$

where $v_{i}^{j}$ is the center of $\left|V_{i}^{j}\right|$.

In the next step we construct chains of covering relations along numerically observed heteroclinic connections. After an extensive numerical simulation we found approximate heteroclinic points for $P$ linking some pairs of periodic points $p_{i}^{j}$ and pair $q_{1}^{12}, q_{2}^{14}$; see Figure 15 . Put

$$
\begin{aligned}
c_{1,2} & =p_{1}^{8}+A_{1}^{8} \cdot(0.00158202,0,0)^{T}, \\
c_{2,1} & =p_{2}^{8}+A_{2}^{8} \cdot(0.00128584,0,0)^{T}, \\
c_{3,4} & =p_{3}^{8}+A_{3}^{8} \cdot(-0.000134682,0,0)^{T}, \\
c_{4,3} & =p_{4}^{16}+A_{4}^{16} \cdot(0.00039344,0,0)^{T}, \\
c_{1,3} & =p_{1}^{8}+A_{1}^{8} \cdot(-0.000884928,0,0)^{T}, \\
h_{1,2} & =q_{1}^{12}+A_{1}^{12} \cdot\left(-8.1098 \cdot 10^{-5}, 0,0\right)^{T}, \\
h_{2,1} & =q_{2}^{14}+A_{2}^{14} \cdot\left(-1.9639 \cdot 10^{-7}, 0.0010592239,0\right)^{T} .
\end{aligned}
$$

These points lie in linear approximations of the unstable manifold at each periodic point. Moreover, from nonrigorous numerical simulations we obtain that these points satisfy

$$
\begin{aligned}
\left\|P^{64}\left(c_{1,2}\right)-p_{2}^{8}\right\|_{1} & \approx 2.176151 \cdot 10^{-4}, \\
\left\|P^{48}\left(c_{2,1}\right)-p_{1}^{8}\right\|_{1} & \approx 0.203890 \cdot 10^{-4}, \\
\left\|P^{88}\left(c_{3,4}\right)-p_{4}^{16}\right\|_{1} & \approx 0.012064 \cdot 10^{-4},
\end{aligned}
$$



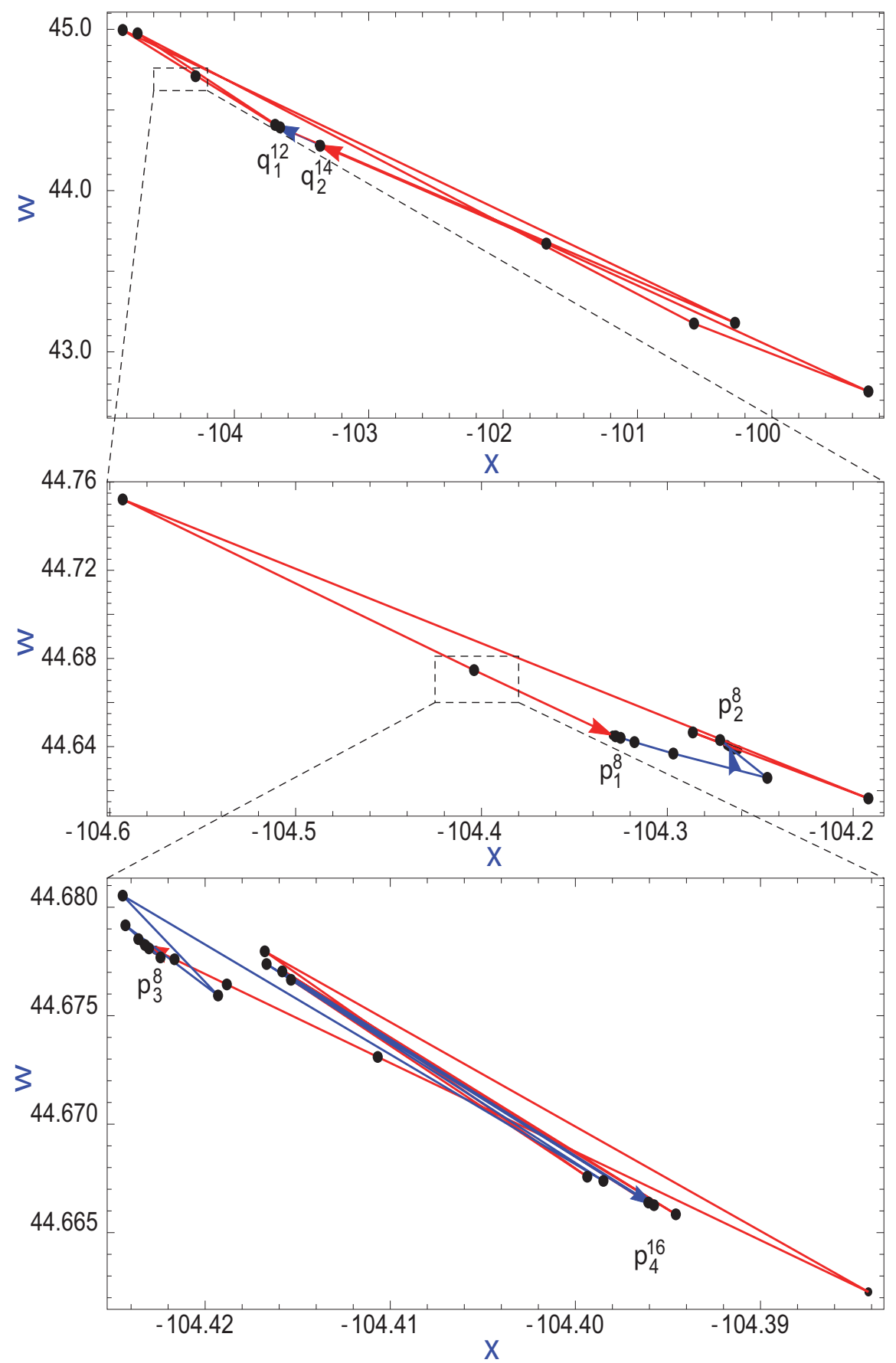

Figure 15. Approximate heteroclinic trajectories of points (top panel) $h_{1,2}$ and $h_{2,1}$, (middle panel) $c_{1,2}$ and $c_{2,1}$, and (bottom panel) $c_{3,4}$ and $c_{4,3}$; see (10). Red and blue are used to distinguish points on two approximate heteroclinic orbits in opposite directions. 


$$
\begin{aligned}
\left\|P^{88}\left(c_{4,3}\right)-p_{3}^{8}\right\|_{1} & \approx 2.123667 \cdot 10^{-4} \\
\left\|P^{64}\left(c_{1,3}\right)-p_{3}^{8}\right\|_{1} & \approx 1.600482 \cdot 10^{-4} \\
\left\|P^{64}\left(h_{1,2}\right)-q_{2}^{14}\right\|_{1} & \approx 7.513020 \cdot 10^{-4} \\
\left\|P^{42}\left(h_{2,1}\right)-q_{1}^{12}\right\|_{1} & \approx 5.470063 \cdot 10^{-4}
\end{aligned}
$$

and therefore their orbits are good candidates for building heteroclinic chains of covering relations between some pairs of $h$-sets centered at periodic points. Before we state the next lemma, let us introduce the notion of chain covering relation.

Definition 5.2. Let $N=\left(h_{N}, u_{N}, s_{N}\right)$ and $M=\left(h_{M}, u_{M}, s_{M}\right)$ be h-sets such that $n=$ $u_{N}+s_{N}=u_{M}+s_{M}$. Let $f: \mathbb{R}^{n} \supset D \rightarrow \mathbb{R}^{n}$ be a continuous map. We say that the $h$-set $N$ chain $f^{r}$-covers the $h$-set $M$, denoted by $N \stackrel{\stackrel{f}{r}_{\sim}^{r}}{\sim} M$, if there are $h$-sets $K_{i}=\left(h_{i}, u_{i}, s_{i}\right)$, $i=1, \ldots, p, p \geq 0$, and positive integers $r_{0}, \ldots, r_{p}$ such that $r_{0}+\cdots+r_{p}=r$ and

$$
N \stackrel{f^{r_{0}}}{\Longrightarrow} K_{1} \stackrel{f^{r_{1}}}{\Longrightarrow} K_{2} \stackrel{f^{r_{3}}}{\Longrightarrow} \cdots \stackrel{f^{r_{p-1}}}{\Longrightarrow} K_{p} \stackrel{f^{r_{p}}}{\Longrightarrow} M
$$

If $p=0$, then this notion coincides with $N \stackrel{f^{r}}{\Longrightarrow} M$.

Now, we can give the next technical result, which establishes the existence of the symbolic dynamics built on approximate heteroclinic orbits (heteroclinic chains) among periodic points with the same number of unstable dimensions.

Lemma 5.3. The following chain covering relations hold true:

$$
\begin{aligned}
& V_{1}^{8} \stackrel{P^{80}}{\rightsquigarrow} V_{2}^{8}, \text { with }\left(r_{0}, \ldots, r_{9}\right)=(8,8,8,8,8,8,8,8,8,8) \text {, } \\
& V_{2}^{8} \stackrel{P^{64}}{\rightsquigarrow} V_{1}^{8}, \text { with }\left(r_{0}, \ldots, r_{7}\right)=(8,8,8,8,8,8,8,8) \text {, } \\
& V_{3}^{8} \stackrel{P^{96}}{\rightsquigarrow} V_{4}^{16}, \text { with }\left(r_{0}, \ldots, r_{11}\right)=(8,8,8,8,8,8,8,8,8,8,8,8) \text {, } \\
& V_{4}^{16} \stackrel{P_{\rightsquigarrow}^{104}}{\rightsquigarrow} V_{3}^{8} \text {, with }\left(r_{0}, \ldots, r_{11}\right)=(16,8,8,8,8,8,8,8,8,8,8,8) \text {, } \\
& V_{1}^{8} \stackrel{P^{80}}{\rightsquigarrow} V_{3}^{8}, \text { with }\left(r_{0}, \ldots, r_{9}\right)=(8,8,8,8,8,8,8,8,8,8) \text {, } \\
& V_{1}^{12} \stackrel{P^{76}}{\rightsquigarrow} V_{2}^{14} \text {, with }\left(r_{0}, \ldots, r_{8}\right)=(12,8,8,8,8,8,8,8,8) \text {, } \\
& V_{2}^{14} \stackrel{P^{56}}{\rightsquigarrow} V_{1}^{12}, \text { with }\left(r_{0}, \ldots, r_{3}\right)=(14,14,14,14) \text {. }
\end{aligned}
$$

Proof. For each chain covering relation $V_{i_{1}}^{j_{1}} \stackrel{P_{\rightsquigarrow}^{r}}{\mapsto} V_{i_{2}}^{j_{2}}$ listed above we constructed a chain of $h$-sets. Each set in the sequence is centered at the point from the approximate trajectory of the point $c_{i_{1}, i_{2}}$ or $h_{i_{1}, i_{2}}$. The coordinate systems for each $h$-set in the sequence have been computed by propagation of the coordinate system at the set $V_{i_{1}}^{j_{1}}$ by the action of $D P^{r_{i}}$ with some orthonormalization procedure. The diameters of sets were adjusted by hand.

In Figures 16 and 17 we show an illustration of covering relations in chains $V_{2}^{8} \stackrel{P^{64}}{\rightsquigarrow} V_{1}^{8}$ (heteroclinic chaotic chain) and $V_{2}^{14} \stackrel{P^{56}}{\rightsquigarrow} V_{1}^{12}$ (heteroclinic hyperchaotic chain), respectively.

Precise definitions of the $h$-sets used in the CAP proof can be found in the accompanying supplementary material (M103920_01.zip [local/web 8.49MB]). 

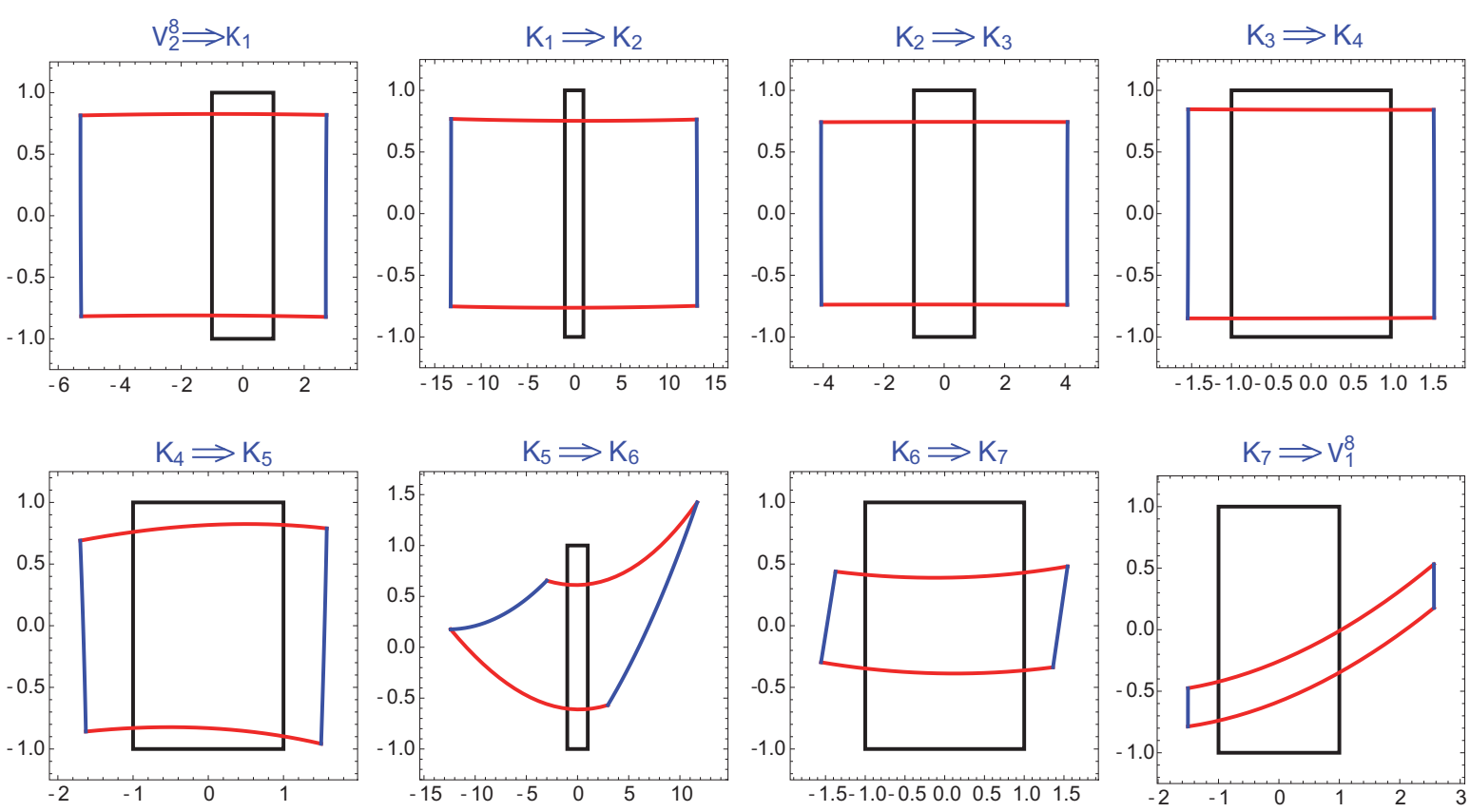

Figure 16. Illustration of the covering relations for $V_{2}^{8} \stackrel{P^{64}}{\rightsquigarrow} V_{1}^{8}$ giving a heteroclinic chaotic chain. The images of h-sets via $P^{8}$ are always shown in a coordinate system of the covered set in which it is the unit ball in the maximum norm. The third dimension is skipped, as it corresponds to very strong contraction.
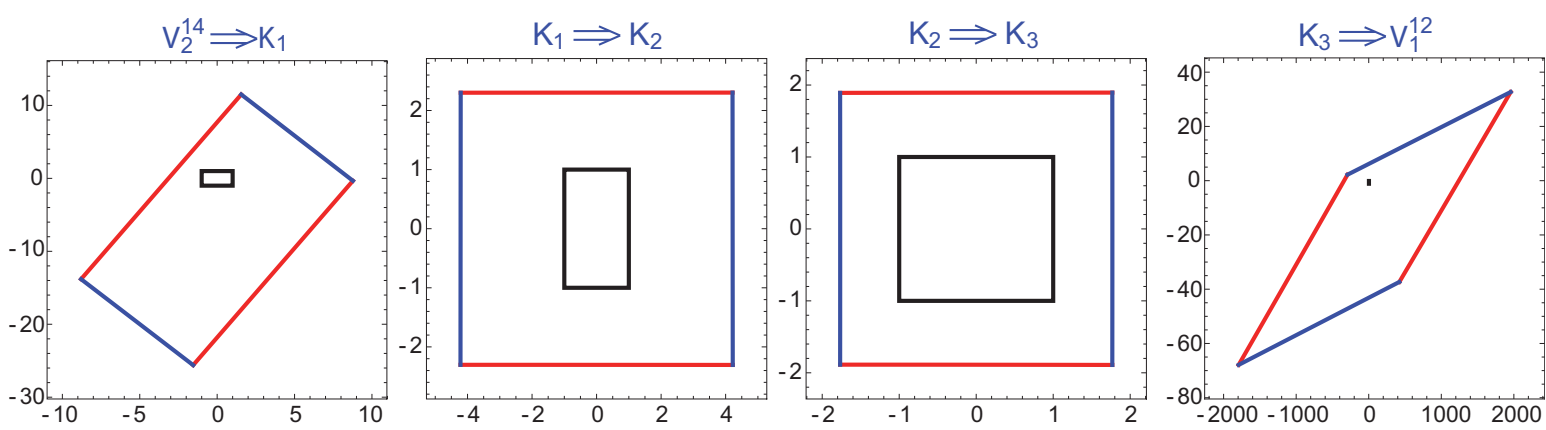

Figure 17. Illustration of the covering relations for $V_{2}^{14} \stackrel{P^{56}}{\rightsquigarrow} V_{1}^{12}$ giving a heteroclinic hyperchaotic chain. The images of h-sets via $P^{14}$ are always shown in a coordinate system of the covered set in which it is the unit ball in the maximum norm. The third dimension is skipped, as it corresponds to very strong contraction.

Remark 5.4. Note that for each chain covering relation in Lemma 5.3 the nominally unstable dimension $u$ and nominally stable dimension $s$ are constant along the sequence. They are equal to $(u, s)=(1,2)$ for $(11)-(15)$ and $(u, s)=(2,1)$ for $(16)-(17)$.

5.2. Dynamical link between hyperchaos and chaos. Another important fact about the existence of the hyperchaotic saddle is whether there is a way to connect the hyperchaotic invariant sets with the chaotic ones, and so initially hyperchaotic orbits will end up being a chaotic orbit. (In fact, the existence of a continuum infinity of such orbits will be shown. 
Clearly not all of them behave like that; we just mention periodic orbits.)

As before, a dynamical link between hyperchaos and chaos is obtained by constructing a chain of covering relations along which the number of nominally unstable directions is changing from 2 to 1 . To this end we take two approximate heteroclinic points

$$
\begin{aligned}
& l_{1,1}=q_{1}^{12}+A_{1}^{12} \cdot\left(-7.0488 \cdot 10^{-5}, 0,0\right)^{T}, \\
& l_{1,4}=q_{1}^{12}+A_{1}^{12} \cdot\left(-6.0427 \cdot 10^{-5}, 0,0\right)^{T},
\end{aligned}
$$

which satisfy

$$
\begin{aligned}
\left\|P^{68}\left(l_{1,1}\right)-p_{1}^{8}\right\|_{1} & \approx 1.285098 \cdot 10^{-4} \\
\left\|P^{52}\left(l_{1,4}\right)-p_{4}^{16}\right\|_{1} & \approx 0.193695 \cdot 10^{-4}
\end{aligned}
$$

Lemma 5.5. The following chain covering relations hold true:

$$
\begin{gathered}
V_{1}^{12} \stackrel{P^{80}}{\rightsquigarrow} V_{1}^{8}, \quad \text { with }\left(r_{0}, \ldots, r_{5}\right)=(12,12,12,12,16,16), \\
V_{1}^{12} \stackrel{P^{64}}{\rightsquigarrow} V_{4}^{16}, \quad \text { with }\left(r_{0}, \ldots, r_{4}\right)=(12,12,12,12,16) .
\end{gathered}
$$

In both cases the change of the nominally unstable dimension from 2 to 1 occurs in the last covering relation in the sequence; see Figure 18 for the illustration of $V_{1}^{12} \stackrel{P^{80}}{\rightsquigarrow} V_{1}^{8}$. The place in the chain where we switch the number of unstable directions is almost arbitrary. This particular choice was our first attempt, and it turned out to work well.

5.3. Cone conditions and heteroclinic orbits. In sections 5.1 and 5.2 we gave some details of the CAP of the first part of Theorem 4.5, which says that every bi-infinite path on the graph shown in Figure 14 is realized by a trajectory of the Poincaré map $P$. This gives us the existence of chaotic and hyperchaotic dynamics for a suitable iterate of the Poincaré map (topological chaotic/hyperchaotic dynamics follow from covering relations). In this subsection the cone conditions [28] are used to prove convergence to periodic orbits and thus the heteroclinic/homoclincic connections among them.

In this subsection we will show that every nonconstant path of the form

$$
\left(v_{0}\right)^{\mathbb{N}}\left(v_{0}, \ldots, v_{n}\right)\left(v_{n}\right)^{\mathbb{N}}
$$

is realized by a heteroclinic/homoclinic connection between periodic orbits $v_{0}$ and $v_{n}$ (that it might be not unique). According to Theorem 3.7 it is enough to specify quadratic forms at each $V_{i}^{j} \in\left\{V_{1}^{8}, V_{2}^{8}, V_{3}^{8}, V_{4}^{16}, V_{1}^{12}, V_{2}^{14}\right\}$ (to give the $h$-sets with cones) and verify that the cone condition is satisfied for each $h$-set. This, however, requires costly $\mathcal{C}^{1}$ computations on large sets and would take a very long time to compute. Instead, for every set $V_{i}^{j}$ we construct a chain $K_{1}, \ldots, K_{p}$ of $h$-sets centered at $v_{i}^{j}$ with the same coordinate system as $V_{i}^{j}$, i.e., $A_{i}^{j}$ defined by (9) but with geometrically decreased diameters. Then we verify that

$$
V_{i}^{j} \stackrel{P^{j}}{\Longrightarrow} K_{1} \stackrel{P^{j}}{\Longrightarrow} \cdots \stackrel{P^{j}}{\Longrightarrow} K_{p-1} \stackrel{P^{j}}{\Longrightarrow} K_{p} \stackrel{P^{j}}{\Longrightarrow} K_{p} \stackrel{P^{j}}{\Longrightarrow} K_{p-1} \stackrel{P^{j}}{\Longrightarrow} \cdots \stackrel{P^{j}}{\Longrightarrow} K_{1} \stackrel{P^{j}}{\Longrightarrow} V_{i}^{j} .
$$



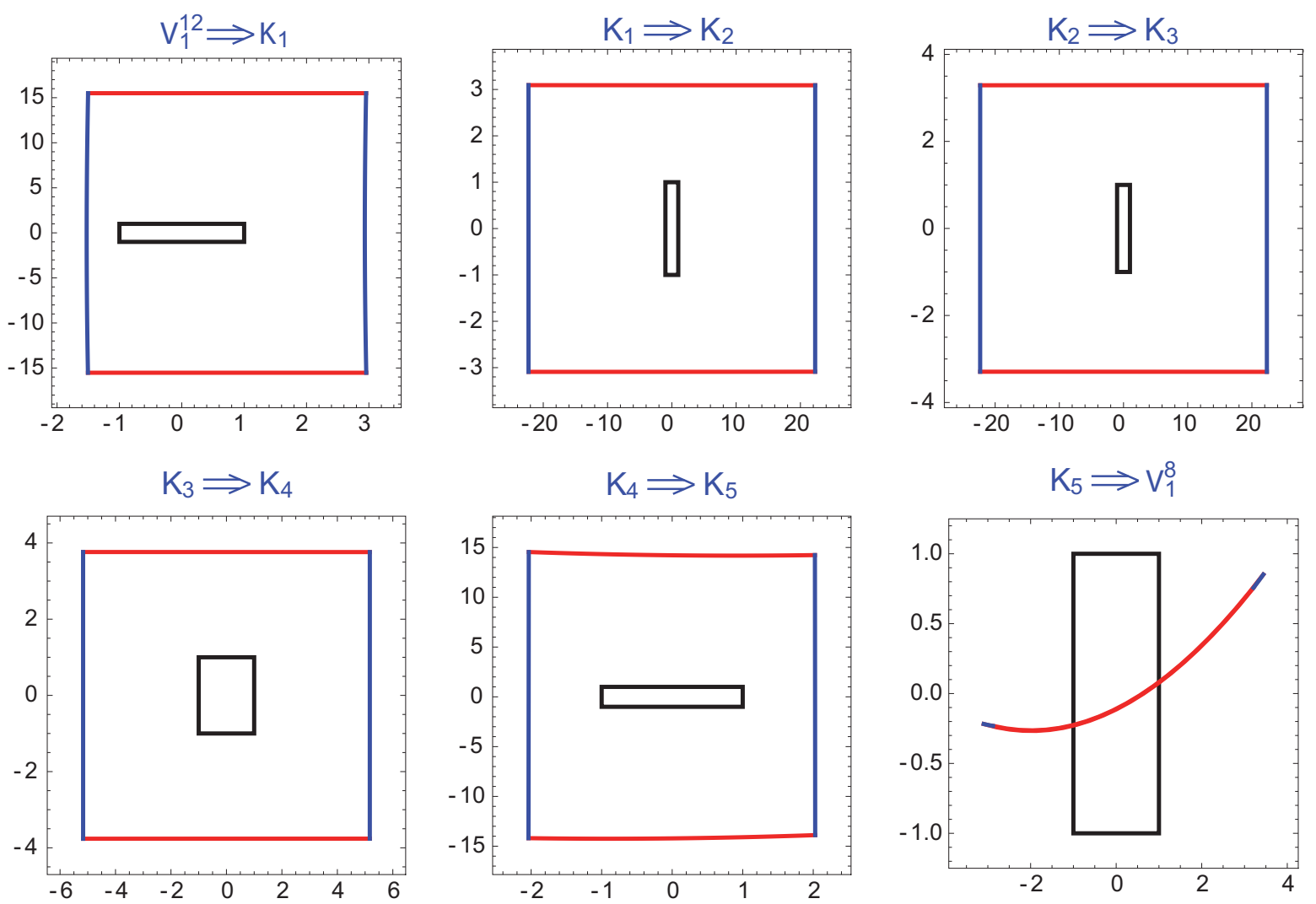

Figure 18. Illustration of the covering relations for $V_{1}^{12} \stackrel{P_{\rightsquigarrow}^{64}}{\rightsquigarrow} V_{1}^{8}$ (heteroclinic hyperchaotic $\rightarrow$ chaotic chain). The change of the number of nominally unstable dimensions from 2 to 1 occurs in the last covering relation in the chain.

Eventually, the cone condition is verified on the smallest set $\left|K_{p}\right|$ for the Poincaré map $P$ expressed in coordinate system $A_{i}^{j}$. In each case the required quadratic form $Q$ is equal to

$$
Q(x, y)=\|x\|^{2}-\|y\|^{2},
$$

and the dimension of $x$ and $y$ is related to the number of nominally unstable and nominally stable dimensions of the corresponding $h$-set $K_{p}$. Thus we have

$$
Q=\operatorname{Diag}(1,-1,-1)
$$

for the points $p_{1}^{8}, p_{2}^{8}, p_{3}^{8}, p_{4}^{16}$ and

$$
Q=\operatorname{Diag}(1,1,-1)
$$

for the points $q_{1}^{12}, q_{2}^{14}$. From Theorem 3.7 it is clear that the numbers of positive and negative coefficients in diagonal matrix $Q$ correspond to the dimensions of the unstable and stable manifolds of the unique periodic point in $\left|K_{p}\right|$, respectively. This periodic point coincides with that resulting from Theorem 4.2 because we can check that the set $\left|K_{p}\right|$ contains the ball $B\left(v_{i}^{j}, 10^{-8}\right)$, which is the distance of the periodic point from its approximation-Theorem 4.2. 
For $x \in\left|K_{p}\right|$ the quadratic form

$$
\left(\left(A_{i}^{j}\right)^{-1} D P^{j}(x) A_{i}^{j}\right)^{T} Q\left(\left(A_{i}^{j}\right)^{-1} D P^{j}(x) A_{i}^{j}\right)-Q
$$

is close to diagonal, as the set $K_{p}$ is very small and the coordinate change $A_{i}^{j}$ almost diagonalizes $D P^{j}\left(v_{i}^{j}\right)$. This is the main reason why we did not normalize columns of matrix $A_{2}^{14}$, the case when a pair of complex eigenvalues occurs. The next step is to use several tools for verification that an interval matrix is positive definite to complete the requirements for covering relations $K_{p} \stackrel{P^{j}}{\Longrightarrow} K_{p}$ with cones (Definition 3.5). In the actual computations we use Rohn's theorem [43] for validation that a symmetric interval matrix is positive definite combined with Gershgorin's theorem and Sylvester's criterion (as the dimension is only 3) [35].

Therefore, now using Theorem 3.7, as all the hypothesis are satisfied, we have proved the main theorem (Theorem 4.5) about heteroclinic/homoclinic connection between periodic orbits.

5.4. Implementation notes. The program that realizes computer-assisted verification of Theorems 4.1, 4.2, and 4.5 has been written by the first author, and the sources are available at his web page [53] and are included in the accompanying supplementary material (M103920_01.zip [local/web 8.49MB]). The program is written in $\mathrm{C}++11$ and has been tested under gcc-4.9.2, gcc-4.9.1, and gcc-4.8.2 on Debian, Ubuntu, and MS Windows 7 operating systems. All time-consuming algorithms are parallelized by means of the OpenMP library supported by modern $\mathrm{C}++$ compilers. The program recognizes the number of cores and tries to use all of them. All the computations were performed on a computer with 64 Intel(R) Xeon(R) CPU E7-8837 2.67GHz cores and 512GB of RAM.

The computations related to Theorem 4.5 took approximately 237 minutes ( 62 for symbolic dynamics and 175 for cone conditions), which gives circa $253 \mathrm{CPU}$ hours in the 64-CPU computer. These computations do not require large memory and in principle can be performed within circa 60 hours on any personal computer equipped with four modern cores.

The computations related to the existence of a trapping region (Theorem 4.1) use quite a large amount of memory (circa 30GB) due to the large number of three-dimensional boxes.

Finally, the verification of the existence and hyperbolicity type of some periodic orbits (Theorem 4.2) takes less than 1 minute on a laptop-type computer.

6. Conclusions. In this paper, we have shown that in high-dimensional systems hyperchaotic behavior is a common feature, forming a global hyperchaotic invariant set, sometimes as a hyperchaotic attractor and sometimes as a saddle invariant, but, in any case, this invariant set can lead to long hyperchaotic transient behavior. The structure of the invariants in this system is shown in Figure 1, where several heteroclinic orbits connect different unstable periodic orbits, some of them with two expanding directions and one of them with just one. This fact generates hyperchaotic and chaotic behaviors. When the attractor is foliated just by chaotic orbits, it can grow when the parameter changes and become tangent to other unstable periodic orbits or some of their invariant manifolds generating new heteroclinic connections and growing in size and, depending on the absorbed invariants, becoming a hyperchaotic attractor. In any case, what we have is that chaotic and hyperchaotic behaviors are always 
present. This global existence of different invariant sets may result in noisy numerical results and great difficulties in distinguishing the type of existing attractor in every region of parameter space. Moreover, the existence of several hyperchaotic sets provides an explanation of the smooth change from chaotic to hyperchaotic attractors due to the appearance of new heteroclinic connections among them, and so the joining of the different sets gives rise to slightly bigger and slightly more hyperchaotic attractors in the sense that the second Lyapunov exponent grows a little.

This coexistence of chaotic and hyperchaotic behaviors is proved via CAP techniques using interval arithmetic and rigorous computing. The proof utilizes the method of covering relations with smooth tools (cone conditions). The proof requires the computation of rigorous bounds for a Poincaré map together with its derivatives. The $\mathrm{C}++$ program which realizes the necessary computations is available from [53] and in the accompanying supplementary material (M103920_01.zip [local/web 8.49MB]).

Acknowledgment. The authors thank their colleague Dr. M. A. Martínez for many interesting discussions and help in the location of unstable periodic orbits.

\section{REFERENCES}

[1] A. Abad, R. BArrio, F. Blesa, And M. Rodriguez, https://sourceforge.net/projects/tidesodes and http://cody.unizar.es/tides.html.

[2] A. Abad, R. Barrio, F. Blesa, and M. Rodríguez, Algorithm 924: TIDES, a Taylor series Integrator for Differential EquationS, ACM Trans. Math. Software, 39 (2012), 5, doi:10.1145/2382585.2382590.

[3] V. S. Afraimovic, V. V. Bykov, And L. P. Shilnikov, The origin and structure of the Lorenz attractor, Soviet Phys. Dokl., 22 (1977), pp. 253-255.

[4] K. T. Alligood, T. Sauer, and J. A. Yorke, Chaos: An Introduction to Dynamical Systems, Textbooks Math. Sci., Springer, New York, 1996, doi:10.1007/b97589.

[5] T. Bakri And F. Verhulst, Bifurcations of quasi-periodic dynamics: Torus breakdown, Z. Angew. Math. Phys., 65 (2014), pp. 1053-1076, doi:10.1007/s00033-013-0363-8.

[6] R. Barrio, F. Blesa, And S. Serrano, Bifurcations and safe regions in open Hamiltonians, New J. Phys., 11 (2009), 053004, doi:10.1088/1367-2630/11/5/053004.

[7] R. BARrio, F. Blesa, AND S. Serrano, Qualitative analysis of the Rössler equations: Bifurcations of limit cycles and chaotic attractors, Phys. D, 238 (2009), pp. 1087-1100, doi:10.1016/j.physd.2009.03.010.

[8] R. Barrio, F. Blesa, And S. Serrano, Topological changes in periodicity hubs of dissipative systems, Phys. Rev. Lett., 108 (2012), 214102, doi:10.1103/PhysRevLett.108.214102.

[9] R. Barrio, F. Blesa, S. Serrano, and A. Shilnikov, Global organization of spiral structures in biparameter space of dissipative systems with Shilnikov saddle-foci, Phys. Rev. E (3), 84 (2011), 035201, doi:10.1103/PhysRevE.84.035201.

[10] R. Barrio, A. Dena, And W. Tucker, A database of rigorous and high-precision periodic orbits of the Lorenz model, Comput. Phys. Commun., 194 (2015), pp. 76-83, doi:10.1016/j.cpc.2015.04.007.

[11] R. Barrio, M. A. Martinez, S. Serrano, And A. Shilnikov, Macro- and micro-chaotic structures in the Hindmarsh-Rose model of bursting neurons, Chaos, 24 (2014), 023128, doi:10.1063/1.4882171.

[12] R. Barrio, M. A. Martínez, S. Serrano, and D. Wilczak, When chaos meets hyperchaos: 4D Rössler model, Phys. Lett. A, 379 (2015), pp. 2300-2305, doi:10.1016/j.physleta.2015.07.035.

[13] L. Basnarkov and L. Kocarev, Forecast improvement in Lorenz-96 system, Nonlinear Process. Geophys., 19 (2012), pp. 569-575, doi:10.5194/npg-19-569-2012.

[14] H. Broer, C. Simó, And R. Vitolo, Bifurcations and strange attractors in the Lorenz-84 climate model with seasonal forcing, Nonlinearity, 15 (2002), pp. 1205-1267, doi:10.1088/0951-7715/15/4/312. 
[15] CAPD, Computer Assisted Proofs in Dynamics: A C++ Package for Rigorous Numerics, http://capd. ii.uj.edu.pl., 2013.

[16] M. P. Dafilis, F. Frascoli, P. J. CAdusch, And D. T. J. Liley, Four dimensional chaos and intermittency in a mesoscopic model of the electroencephalogram, Chaos, 23 (2013), 023111, doi:10.1063/1.4804176.

[17] M. Dhamala And Y.-C. Lai, Unstable periodic orbits and the natural measure of nonhyperbolic chaotic saddles, Phys. Rev. E (3), 60 (1999), pp. 6176-6179, doi:10.1103/physreve.60.6176.

[18] H. R. Dullin And A. Wittek, Complete Poincare sections and tangent sets, J. Phys. A, 28 (1995), pp. 7157-7180, doi:10.1088/0305-4470/28/24/015

[19] M. Eiswirth, Th.-M. Kruel, G. Ertl, and F. W. Schneider, Hyperchaos in a chemical reaction, Chem. Phys. Lett., 193 (1992), pp. 305-310, doi:10.1016/0009-2614(92)85672-w .

[20] N. Fenichel, Persistence and smoothness of invariant manifolds for flows, Indiana Univ. Math. J., 21 (1972), pp. 193-226, doi:10.1512/iumj.1972.21.21017.

[21] P. Frederickson, J. L. Kaplan, E. D. Yorke, and J. A. Yorke, The Liapunov dimension of strange attractors, J. Differential Equations, 49 (1983), pp. 185-207, doi:10.1016/0022-0396(83)90011-6.

[22] T. Gao And Z. Chen, A new image encryption algorithm based on hyper-chaos, Phys. Lett. A, 372 (2008), pp. 394-400, doi:10.1016/j.physleta.2007.07.040.

[23] M. Gidea And C. Robinson, Topologically crossing heteroclinic connections to invariant tori, J. Differential Equations, 193 (2003), pp. 49-74, doi:10.1016/s0022-0396(03)00065-2.

[24] J. Guchenheimer and R. F. Williams, Structural stability of Lorenz attractors, Inst. Hautes Études Sci. Publ. Math., 50 (1979), pp. 59-72, doi:10.1007/bf02684769.

[25] K. KANEKO, Transition from torus to chaos accompanied by frequency lockings with symmetry breaking, Progr. Theoret. Phys., 69 (1983), pp. 1427-1442, doi:10.1143/ptp.69.1427.

[26] T. Kapitaniak, Y. Maistrenko, and S. Popovych, Chaos-hyperchaos transition, Phys. Rev. E (3), 62 (2000), pp. 1972-1976, doi:10.1103/physreve.62.1972.

[27] U. Kirchgraber And D. Stoffer, Possible chaotic motion of comets in the Sun-Jupiter system-a computer-assisted approach based on shadowing, Nonlinearity, 17 (2004), pp. 281-300, doi:10.1088/0951-7715/17/1/016.

[28] H. Kokubu, D. Wilczak, And P. ZGliczyński, Rigorous verification of cocoon bifurcations in the Michelson system, Nonlinearity, 20 (2007), pp. 2147-2174, doi:10.1088/0951-7715/20/9/008.

[29] A. P. Kuznetsov And Y. V. Sedova, Coupled Systems with Hyperchaos and Quasiperiodicity, preprint, arXiv:1504.00811, 2015.

[30] Y. C. Lai, C. Grebogi, J. A. Yorke, And I. Kan, How often are chaotic saddles nonhyperbolic?, Nonlinearity, 6 (1993), pp. 779-797, doi:10.1088/0951-7715/6/5/007.

[31] Y.-C. Lai And T. TÉL, Transient Chaos, Appl. Math. Sci. 173, Springer, New York, 2011, doi:10.1007/978-1-4419-6987-3.

[32] Q. LI, A topological horseshoe in the hyperchaotic Rössler attractor, Phys. Lett. A, 372 (2008), pp. 29892994, doi:10.1016/j.physleta.2007.11.071.

[33] Q. LI, S. TANG, AND X.-S. YANG, Hyperchaotic set in continuous chaos-hyperchaos transition, Commun. Nonlinear Sci. Numer. Simul., 19 (2014), pp. 3718-3734, doi:10.1016/j.cnsns.2014.03.001.

[34] T. Matsumoto, L. O. Chua, And K. Kobayashi, Hyperchaos: Laboratory experiment and numerical confirmation, IEEE Trans. Circuits and Systems, 33 (1986), pp. 1143-1149, doi:10.1109/tcs.1986.1085862.

[35] C. D. Meyer, Matrix Analysis and Applied Linear Algebra, SIAM, Philadelphia, 2000.

[36] K. Mischaikow And M. Mrozek, Chaos in Lorenz equations: A computer assisted proof, Bull. Amer. Math. Soc. (N.S.), 33 (1995), pp. 66-72, doi:10.1090/s0025-5718-98-00945-4.

[37] A. Neumaier, Interval Methods for Systems of Equations, Cambridge University Press, Cambridge, UK, 1991.

[38] R. F. Pereira, S. E. De S. Pinto, R. L. Viana, S. R. Lopes, and C. Grebogi, Periodic orbit analysis at the onset of the unstable dimension variability and at the blowout bifurcation, Chaos, 17 (2007), 023131, doi:10.1063/1.2748619.

[39] P. PilarczyK, Computer assisted proof of the existence of a periodic orbit in the Rössler equations, in Proceedings of the International Conference on Differential Equations (Berlin, 1999), World Scientific, Singapore, 2000, pp. 228-230, doi:10.1142/9789812792617_0047. 
[40] P. Pilarczyk, Topological numerical approach to the existence of periodic trajectories in ODE's, in Discrete and Continuous Dynamical Systems, A Supplement Volume: Dynamical Systems and Differential Equations, AIMS, Springfield, MO, 2003, pp. 701-708, doi:10.3934/proc.2003.2003.701.

[41] J. Rasmussen, E. Mosekilde, and C. Reick, Bifurcations in two coupled Rössler systems, Math. Comput. Simulation, 40 (1996), pp. 247-270, doi:10.1016/0378-4754(95)00036-4.

[42] P. C. RECH, Chaos and hyperchaos in a Hopfield neural network, Neurocomputing, 74 (2011), pp. 33613364, doi:10.1016/j.neucom.2011.05.016.

[43] J. Rohn, Positive definiteness and stability of interval matrices, SIAM J. Matrix Anal. Appl., 15 (1994), pp. 175-184, doi:10.1137/S0895479891219216.

[44] O. E. RössleR, An equation for hyperchaos, Phys. Lett. A, 71 (1979), pp. 155-157, doi:10.1016/0375-9601(79)90150-6.

[45] O. E. RössleR, An equation for continuous chaos, Phys. Lett. A, 57 (1976), pp. 397-398, doi:10.1016/0375-9601(76)90101-8.

[46] R. Stoop, J. Peinke, J. Parisi, B. Röhricht, and R. P. Huebener, A p-Ge semiconductor experiment showing chaos and hyperchaos, Phys. D, 35 (1989), pp. 425-435, doi:10.1016/0167-2789(89)90078-X.

[47] J.-G. Tong, Z.-X. Zhang, Q.-L. Sun, And Z.-Q. Chen, Design of wireless sensor network node with hyperchaos encryption based on FPGA, in Proceedings of the International Workshop on ChaosFractals Theories and Applications (IWCFTA '09), 2009, pp. 190-194, doi:10.1109/IWCFTA.2009.47.

[48] W. Tucker, A rigorous ODE solver and Smale's 14th problem, Found. Comput. Math., 2 (2002), pp. 53117, doi:10.1007/s002080010018.

[49] R. Vitolo, P. Glendinning, And J. A. C. Gallas, Global structure of periodicity hubs in Lyapunov phase diagrams of dissipative flows, Phys. Rev. E (1), 84 (2011), 016216, doi:10.1103/PhysRevE.84.016216.

[50] I. Walawska ANd D. Wilczak, $c^{1}-H O-$ An Implicit Algorithm for Validated Enclosures of the Solutions to Variational Equations for ODEs, preprint, arXiv:1509.07388, 2015.

[51] F. Waldner and R. Klages, Symmetric Jacobian for local Lyapunov exponents and Lyapunov stability analysis revisited, Chaos Solitons Fractals, 45 (2012), pp. 325-340, doi:10.1016/j.chaos.2011.12.014.

[52] S. Wiggins, Normally Hyperbolic Invariant Manifolds in Dynamical Systems, Appl. Math. Sci. 105, Springer, New York, 1994, doi:10.1007/978-1-4612-4312-0.

[53] D. WilczaK, http://www.ii.uj.edu.pl/ wilczak.

[54] D. WilczaK, The existence of Shilnikov homoclinic orbits in the Michelson system: A computer assisted proof, Found. Comput. Math., 6 (2006), pp. 495-535, doi:10.1007/s10208-005-0201-2.

[55] D. Wilczak, Abundance of heteroclinic and homoclinic orbits for the hyperchaotic Rössler system, Discrete Contin. Dyn. Syst. Ser. B, 11 (2009), pp. 1039-1055, doi:10.3934/dcdsb.2009.11.1039.

[56] D. Wilczak, Uniformly hyperbolic attractor of the Smale-Williams type for a Poincaré map in the Kuznetsov system, SIAM J. Appl. Dyn. Syst., 9 (2010), pp. 1263-1283, doi:10.1137/100795176.

[57] D. Wilczak and P. Zgliczyński, Period doubling in the Rössler system-a computer assisted proof, Found. Comput. Math., 9 (2009), pp. 611-649, doi:10.1007/s10208-009-9040-x.

[58] A. Wolf, J. B. Swift, H. L. Swinney, And J. A. Vastano, Determining Lyapunov exponents from a time series, Phys. D, 16 (1985), pp. 285-317, doi:10.1016/0167-2789(85)90011-9.

[59] S. YAnChuK AND T. KAPITANiaK, Chaos-hyperchaos transition in coupled Rössler systems, Phys. Lett. A, 290 (2001), pp. 139-144, doi:10.1016/S0375-9601(01)00651-X.

[60] P. ZgLiczyński, Computer assisted proof of chaos in the Rössler equations and in the Hénon map, Nonlinearity, 10 (1997), pp. 243-252, doi:10.1088/0951-7715/10/1/016

[61] P. Zgliczyński, $C^{1}$-Lohner algorithm, Found. Comput. Math., 2 (2002), pp. 429-465, doi:10.1007/s102080010025.

[62] P. ZGLicZyńsKi, Covering relations, cone conditions and the stable manifold theorem, J. Differential Equations, 246 (2009), pp. 1774-1819, doi:10.1016/j.jde.2008.12.019.

[63] P. ZGLiczyóski And M. Gidea, Covering relations for multidimensional dynamical systems, J. Differential Equations, 202 (2004), pp. 32-58, doi:10.1016/j.jde.2004.03.013. 